\title{
On-Orbit Calibration of Photodiodes for Attitude Determination
}

\author{
John C. Springmann* and James W. Cutler \\ University of Michigan, Ann Arbor, Michigan 48109
}

DOI: $10.2514 / 1 . G 000175$

\begin{abstract}
A method is presented for the on-orbit calibration of photodiodes for sun sensing in an attitude determination system. The calibration estimates the scale factors and alignment angles of the photodiodes, resulting in a higher attitude determination accuracy than achieved with the preflight calibration parameters. The calibration is accomplished through the simultaneous estimation of the spacecraft attitude and calibration parameters. This approach, as opposed to an attitude-independent method, enables the calibration of an arbitrary number of photodiodes mounted in any orientation on the spacecraft and facilitates the use of an attitude-dependent Earth albedo model. The method is formulated within both an extended Kalman filter and an unscented filter. The filters are demonstrated by application to flight data from the Radio Aurora Explorer satellites and result in an average angular improvement of $10 \mathrm{deg}$ in sun vector measurements with the photodiodes. Attitude determination accuracies of below $1 \mathrm{deg}$ in each axis are demonstrated using the calibrated photodiodes in combination with a low-cost three-axis magnetometer and rate gyroscope.
\end{abstract}

\section{Introduction}

$\mathbf{S}$ UN sensors are the most widely used sensor type in attitude determination systems [1]. They are used to measure the vector from the spacecraft to the sun (herein referred to as the sun vector) in the spacecraft body-fixed frame, and their angular accuracies range from several degrees to less than an arcsecond. Photodiodes, which generate current as a function of incoming light [2], are the most basic type of sun sensor. A standalone photodiode provides a measurement of the angle between the sun vector and the direction normal to the photosensitive plane, effectively measuring one component of the sun vector. For multi-axis sun sensing with photodiodes, two common schemes exist: 1) individual photodiodes can be mounted in different orientations, either within a single sensor package [3] or distributed over the spacecraft body [4], for up to three-component sun sensing, or 2) multiple photodiodes and a mask can be combined within a single sensor package for two-axis sun sensing. The angular accuracy of the former scheme is on the order of degrees, and the angular accuracy of the latter, which can be as good as arcseconds, depends on the complexity of the sensor [5,6]. A photodiode-based sensor with accuracy on the order of degrees is typically referred to as a coarse sun sensor, whereas sensors with angular accuracies on the order of tenths of degrees or better are referred to as fine sun sensors. Fine sun sensors commonly use more advanced components than photodiodes, such as CMOS sensors [7].

This work focuses on coarse sun sensors composed of individual photodiodes mounted at different angles. This photodiode scheme is extremely common on CubeSats and other small spacecraft [8-12] because of its simplicity and low cost. One configuration that is typical of CubeSats is to mount the photodiodes orthogonal to each other by placing one on each surface of the spacecraft $[8,9,12]$. However, this configuration does not provide three-component sun sensing in all directions because the conical field of view of

Received 24 July 2013; revision received 14 October 2013; accepted for publication 15 October 2013; published online 29 April 2014. Copyright $@$ 2013 by John C. Springmann and James W. Cutler. Published by the American Institute of Aeronautics and Astronautics, Inc., with permission. Copies of this paper may be made for personal or internal use, on condition that the copier pay the $\$ 10.00$ per-copy fee to the Copyright Clearance Center, Inc., 222 Rosewood Drive, Danvers, MA 01923; include the code 1533-3884/14 and $\$ 10.00$ in correspondence with the CCC.

*Ph.D. Candidate, Department of Aerospace Engineering; jspringm@ umich.edu.

${ }^{\dagger}$ Assistant Professor, Department of Aerospace Engineering; jwcutler@ umich.edu.

‡Osram SFH 2430 photodiodes, which flew on the RAX satellites [9], can be purchased for $\$ 1.84$ per sensor; see http://www.digikey.com/ product-detail/en/SFH\%202430-Z/475-2579-1-ND/1228076 [retrieved 1 March 2013]. photodiodes is typically less than $180 \mathrm{deg}$ [9]. A natural improvement to this is to use additional photodiodes mounted in various orientations over the spacecraft body to achieve three-component sun sensing in all directions. In addition to enabling three-component sun sensing, this configuration provides more information for the attitude determination system, resulting in a potential angular accuracy improvement. This photodiode scheme has been used on various spacecraft $[\underline{3}, \underline{10}, \underline{13}]$, and a design method to determine optimal photodiode orientations for sun sensing is given in [4].

There are two parameters critical for accurate sun sensing with photodiodes: the scale factor, which relates the measured current to the sun vector component, and the orientation of each photodiode on the spacecraft. The scale factor is dependent on the characteristics of both the photodiode and surrounding circuitry, and it will be discussed further in Sec. III.A. Although the scale factor can be estimated from preflight calibration, photodiodes are known to degrade on orbit due to radiation, and previous flight experience demonstrates that this has a significant effect on the scale factor [14]. Additionally, thorough preflight calibration requires a light source that is calibrated to match the characteristics of sunlight in orbit. Onorbit estimation of the scale factor provides the best estimate of the on-orbit sensor characteristics and lowers the spacecraft development time and cost by mitigating rigorous preflight calibration requirements. Similarly, the preflight orientation of the photodiodes is known if sufficiently high tolerance procedures are used during spacecraft integration, but any high tolerance procedures that add cost to the integration defeat the low-cost benefits of photodiodes, and the orientation may change during the launch regardless of the initial tolerance. Therefore, the photodiode orientation is also estimated in flight.

The contributions of this work are the development and application of a new, on-orbit photodiode calibration method to estimate the photodiode scale factor and orientation. Implementations of the calibration within both an extended Kalman filter (EKF) and unscented filter (UF) are presented and compared. This recursive, attitude-dependent approach enables the inclusion of an Earth albedo model, which is demonstrated to be an important aspect of the calibration. In the application to flight data from the Radio Aurora Explorer (RAX) satellites, an average of a $10 \mathrm{deg}$ improvement in accuracy of the photodiode-based sun vector measurement is achieved with the on-orbit calibration. In a previous conference paper [15], only the EKF-based formulation was discussed, and the filter was applied to a limited amount of flight data. In this paper, both the UF- and EKF-based approaches are presented, a simulated comparison is discussed, and the filters are applied to a broader set of flight data.

The remainder of this paper is organized as follows. Existing calibration techniques and the motivation for formulating the photo- 
diode calibration as a recursive attitude-dependent method is discussed in Sec. II. The formulation of the calibration within both the EKF and UF is discussed in Sec. III, and both filters are compared via simulation and applied to flight data in Sec. IV. The impact on the sun vector angular accuracy and the importance of including the Earth albedo model is also discussed in Sec. IV. A discussion of some of the assumptions and techniques used in the calibration is presented in Sec. $\underline{\mathrm{V}}$, and conclusions are given in Sec. VI.

\section{Existing Calibration Techniques}

In general, sensor measurements are corrupted by errors such as scale factors, bias, and angular misalignments. Calibration is used to estimate and subsequently compensate for the sensor errors, and it is critical for accurate attitude estimation. Many calibration techniques have been presented in the literature; this section is not meant to include an exhaustive overview of existing calibration techniques, but rather to summarize the most relevant types of methods in the context of the new photodiode calibration presented in this paper.

The calibration process consists of modeling the sensor of interest and estimating the parameters of the model using sensor measurements from either ground-based testing or on-orbit operations. Calibration using only on-orbit data is referred to as on-orbit calibration, and it is advantageous over ground-based calibration because it mitigates the time and resources required for preflight calibration and accounts for changes to sensor characteristics once on orbit.

On-orbit calibration techniques can be categorized as either attitude independent or attitude dependent. Attitude-independent calibration does not require attitude knowledge and is accomplished by minimizing a scalar objective function that is dependent on the calibration parameters. Typical scalar objective functions are the angle between two vectors in the same frame or the magnitude of a vector, with examples of these approaches found in [16-20]. Both of these attitude-independent methods can be applied to various types of three-axis sensors. Attitude-dependent techniques are recursive methods that use attitude estimates for the sensor calibration, and thus simultaneously estimate attitude and sensor calibration parameters. Examples of this approach include [21] and [22], which use an extended Kalman filter and an unscented filter, respectively, to estimate sensor misalignments, rate gyroscope scaling and bias, and attitude.

Regarding sun sensors specifically, on-orbit calibration of coarse sensors is not prevalent in the literature. Furthermore, the models of fine sun sensors are dependent on the sensor design, so existing calibration methods are typically applicable to only specific sensors $[5,6]$. While both the attitude-independent and attitude-dependent calibration approaches mentioned previously can be applied to onorbit sun sensor calibration, the direct application of these methods requires a sun sensor that provides a three-component sun vector measurement $[23,24]$. Since photodiodes provide a measurement of a single sun vector component, a three-component measurement is not always available, making the application of these methods nontrivial.

The new, on-orbit calibration method presented in this paper has been developed for standalone photodiodes in any orientation and does not require the simultaneous illumination of multiple sensors, which would be required for a three-component vector measurement. This enables the calibration of an arbitrary number of illuminated sensors and facilitates the use of an attitude-dependent Earth albedo model. Earth albedo is the reflection of sunlight from Earth's surface, and it can significantly degrade the accuracy of photodiode-based sun sensing. Both of these aspects are discussed in more detail with the calibration methodology in the next section.

\section{Formulation of Attitude Estimation and Calibration Filter}

On-orbit photodiode calibration is achieved by estimating the calibration parameters and spacecraft attitude simultaneously. We formulate the estimation problem by extending existing recursive attitude estimation methods to include the calibration parameters as states, which is a similar approach to that of existing attitudedependent on-orbit calibration techniques that deal with star tracker and rate gyroscope misalignment $[21,22]$. The calibration has been implemented within both an EKF and $\overline{\mathrm{UF}}$. The EKF is a widely used approach for nonlinear state estimation and has become a standard method for satellite attitude determination $[25,26]$. The UF is a more recent estimation method that uses a different propagation technique than the standard EKF [27], and it can be used as an alternative, potentially more accurate estimator for attitude estimation [26,28]. In general, UFs can be advantageous over the standard EKF because the expected error is lower, they can be applied to nondifferentiable functions, Jacobian matrices are not required, and the UF is valid to higher-order expansions than the standard EKF [28]. But the degree of improvement over the EKF depends on the nature of estimation problem, and UFs are more computationally intensive than the EKF, particularly if parallel processing is not available. A comparison of the photodiode calibration accuracy when using an EKF and an UF is discussed in Sec. IV.B.

Direct application of both the EKF and UF to attitude estimation is nontrivial due to the orthogonality constraint of the attitude matrix, or equivalently, the unity norm constraint when quaternions are used to parameterize attitude; the norm constraint can be violated by the linear measurement updates of the standard EKF. This shortfall has been widely studied, and a common implementation of an EKF for attitude estimation has become known as the multiplicative EKF (MEKF). The term multiplicative stems from the fact that quaternion multiplication supplements the standard additive state update within the EKF $[25,29]$. The multiplicative approach can also be used to satisfy the quaternion norm constraint in unscented filtering, and the specific implementation of this UF for attitude estimation is called the unscented quaternion estimator (USQUE) [28]. The photodiode calibration is accomplished by extending the MEKF and USQUE for the estimation of the calibration parameters along with spacecraft attitude.

The remainder of this section is organized as follows. The photodiode measurement model and Earth albedo are discussed in Sec. III.A. This provides the foundation for extending the MEKF and USQ $\overline{\mathrm{UE}}$ to include the photodiode calibration, which is presented in Secs. III.B and III.C. While the derivation of the MEKF and USQUE are beyond the scope of this text, the complete equations for each are given in the Appendix and will be referenced in this section.

\section{A. Photodiode Measurement Model}

The foundation of the inclusion of photodiode measurements in recursive estimation is their measurement model. The measurement model for a photodiode illuminated by only the sun is given by Eq. (1), where $\tilde{I}$ is the measured current output; $E_{\mathrm{AM} 0}$ is the irradiance of the sunlight $\underline{\S} ; I_{0}$ is the maximum current output of the photodiode; $E_{\text {cal }}$ is a scaling parameter that relates the current output to the incident irradiance and is dependent on the specific photodiode and surrounding circuitry; $\boldsymbol{n}$ is the $3 \times 1$ unit vector that defines the direction normal to the photosensitive plane, herein referred to as the photodiode normal direction; $s$ is the $3 \times 1$ unit sun vector; and $\eta$ is the zero mean Gaussian measurement noise []]. Even though both $\boldsymbol{n}$ and $s$ are unit vectors, they are not denoted with the common unit vector notation of $\hat{\boldsymbol{n}}$ and $\hat{\boldsymbol{s}}$ because the hat symbol is reserved to denote estimated quantities.

$$
\tilde{I}=\frac{E_{\mathrm{AM} 0} I_{0}}{E_{\mathrm{cal}}} \boldsymbol{n}^{T} \boldsymbol{s}+\eta
$$

The quantity $\boldsymbol{n}^{T} \boldsymbol{s}$ is equivalent to $\cos (\theta)$, where $\theta$ is the angle between the two vectors. Instead of $\boldsymbol{n}^{T} \boldsymbol{s}, \cos (\theta)$ is commonly used in photodiode models, and photodiodes are sometimes referred to as cosine detectors [1]. Phototiodes generate current anytime $0<\theta \leq$ $90 \mathrm{deg}$, but the output deviates from the cosine model of Eq. (1) at high angles. The photodiode field of view (FOV) is the conical region

${ }^{\S}$ The subscript AM0 denotes air mass zero, meaning zero atmospheres. $E_{\mathrm{AM} 0}$ is the solar irradiance at $1 \mathrm{AU}$ with no loss due to Earth's atmosphere. 
over which the cosine model is valid. The exact FOV varies by sensor, but a half-angle of 60-70 deg is typical.

Photodiodes in low Earth orbit are also subject to illumination from sunlight reflected by Earth, which is called Earth albedo [30]. The irradiance of Earth albedo can be up to $30-40 \%$ of the solar irradiance, resulting in a potentially significant degradation of a sun vector measurement if it is not accounted for. Albedo is typically treated as noise in attitude estimation, and this approach can be successful particularly when other attitude sensors are available to complement the photodiodes. But we have found that this approach of simply increasing the measurement covariance is not sufficient for photodiode calibration because the resulting state-error covariance of the calibration parameters is too high; the confidence in the calibration parameter estimates is not a significant improvement over the precalibration state estimates. Therefore, we include a model of albedo in the filter to remove its contribution from the measurements. The albedo model used was developed by Bhanderi $[8,30,31]$ using Earth reflectivity measurements from NASA's Total Ozone Mapping Spectrometer (TOMS), and the model has been verified through comparison to flight data from the $\oslash$ rstead satellite. The photodiode measurement model with the inclusion of Earth albedo is

$$
\tilde{I}=\frac{I_{0}}{E_{\mathrm{cal}}}\left(E_{\mathrm{AM} 0} \boldsymbol{n}^{T} \boldsymbol{s}+E_{a}\right)+\eta
$$

where the albedo irradiance $E_{a}$ is given by Eq. (3).

Earth albedo is a function of the relative positions of the sun, Earth, and satellite, and the Earth reflectivity varies with longitude, latitude, and atmospheric conditions such as cloud coverage. To account for the varying reflectivity in the albedo model, Earth's surface is partitioned into cells, and the reflectivity of each cell is the average of measurements over a 1-year time period. The latitude and longitude of the center of each cell is denoted by $\left(\phi_{g}, \theta_{g}\right) \in D$, where $D$ is the set of all cell locations. The incident Earth albedo irradiance on a photodiode with normal direction $\boldsymbol{n}$ is

$$
E_{a}=\sum_{V_{\mathrm{sun}} \cap V_{\mathrm{sat}}} E_{c}\left(\phi_{g}, \theta_{g}\right) \boldsymbol{n}^{T} \boldsymbol{r}_{g}
$$

where $V_{\text {sun }} \subset D$ and $V_{\text {sat }} \subset D$ are the cells in the field of view of the sun and satellite, respectively; $E_{c}$ is the irradiance reflected by the $g$ th cell in the direction of $\boldsymbol{r}_{g}$, which is a function of the reflectivity of the cell, direction of incoming solar irradiance, and direction to the satellite; and $\boldsymbol{r}_{g}$ is the vector from the satellite to the center of the cell. In this work, the average Earth reflectivity data from the year 2005 is used for the Earth albedo model, which is the latest available TOMS data. A map of the average reflectivity over the 1-year duration is shown in Fig. 1a, and the standard deviation of those measurements is shown in Fig. $\overline{1 b}$. Although the reflectivity is nearly $100 \%$ over some portions of Earth, the albedo is dependent on the reflectivity as well as the angle of incoming sunlight, and the maximum albedo over the poles is between $30 \%$ and $40 \%$ of direct sun irradiance. Further details of the albedo model development and calculation of $E_{c}$ can be found in the existing literature [ $\underline{8}, 31]$. It is not written explicitly, but $E_{a}$ is a function of spacecraft attitude; the attitude is required to compute $\boldsymbol{n}$ and $\boldsymbol{r}_{g}$ in the same frame for Eq. (3).

The information available from a photodiode measurement for attitude determination is the sun vector component along the photodiode normal direction, $\boldsymbol{n}^{T} \boldsymbol{s}$. This quantity can also be written as a function of spacecraft attitude and the sun vector in the inertial reference frame, which is a known function of time and satellite position. This is given by Eq. (4), where $\left.\right|_{B}$ indicates that the preceding quantity is resolved in the satellite body-fixed frame, $\left.\right|_{R}$ indicates that the quantity is resolved in the inertial reference frame, and $A$ is the $3 \times 3$ proper orthogonal attitude matrix defining the orientation of the body-fixed frame relative to the reference frame. The dependence of the photodiode normal direction and spacecraft attitude on the calculation of Earth albedo has also been noted explicitly in Eq. (므).

$$
\left.\left(\boldsymbol{n}^{T} \boldsymbol{s}\right)\right|_{B}=\left.\left.\boldsymbol{n}^{T}\right|_{B} A \boldsymbol{s}\right|_{R}
$$

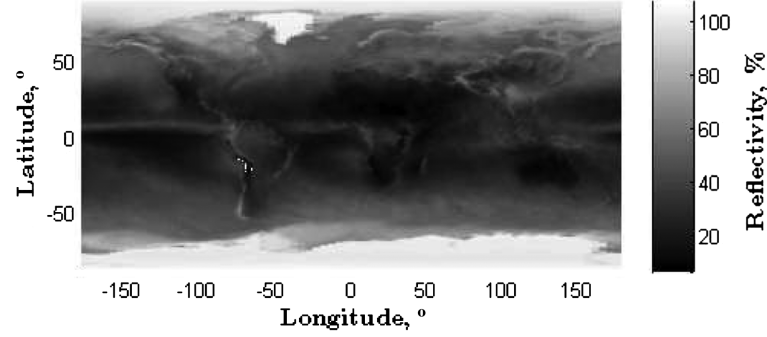

a) Mean

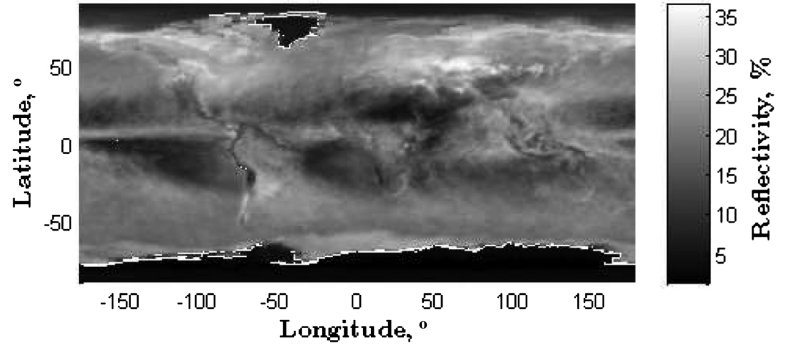

b) Standard deviation

Fig. 1 The mean and standard deviation of Earth reflectivity measurements.

The measurement model that relates the photodiode measurement to spacecraft attitude [analagous in Eqs. (A7) and (A35) of the MEKF and USQUE] is obtained by substituting Eq. (ㄴ) into Eq. (2). The result is given by Eq. (5), where the $j$ subscripts indicate that the terms are specific to the $j$ th photodiode and the substitution $C_{j}=$ $E_{\mathrm{AM} 0} I_{0, j} / E_{\mathrm{cal}, j}$ has been used. $C_{j}$ is the sensor scale factor that will be estimated with on-orbit calibration. It is a dimensional scale factor that is equivalent to the maximum current output caused by irradiance of only direct sunlight.

$$
\tilde{I}_{j}=\left.\left.C_{j} \boldsymbol{n}_{j}^{T}\right|_{B} A \boldsymbol{s}\right|_{R}+C_{j} \frac{E_{a, j}\left(\boldsymbol{n}_{j}, A\right)}{E_{\mathrm{AM} 0}}+\eta
$$

As discussed in Sec. I, $C_{j}$ and $\boldsymbol{n}_{j}$ in Eq. (5) are critical for accurate attitude determination and are the calibration parameters that will be estimated.

\section{B. Integration into MEKF and USQUE}

With the photodiode measurement model in hand, the MEKF and USQUE are extended to include the photodiode calibration. The MEKF and USQUE estimate six states: the $3 \times 1$ attitude error vector $\delta \boldsymbol{p}$, which in combination with quaternions being propagated within the filter quantifies spacecraft attitude, and the $3 \times 1$ rate gyroscope bias $\boldsymbol{\beta}$ (see the Appendix). For photodiode calibration, we use these same states and include the calibration parameters as additional states. The photodiode normal directions $\boldsymbol{n}_{j}$ are parameterized by their corresponding azimuth and elevation, $\alpha_{j}$ and $\epsilon_{j}$, respectively. This is given by Eq. (6) (there is an observability problem with this parameterization that is discussed in Sec. III.C).

$$
\boldsymbol{n}_{j}=\left[\begin{array}{lll}
\cos \left(\epsilon_{j}\right) \cos \left(\alpha_{j}\right) & \cos \left(\epsilon_{j}\right) \sin \left(\alpha_{j}\right) & \sin \left(\epsilon_{j}\right)
\end{array}\right]^{T}
$$

The azimuth and elevation are used rather than the three-component normal vector because three components of a unit vector are not linearly independent. The full state vector is then

$$
\boldsymbol{x}_{\left(6+3 m_{p}\right) \times 1}=\left[\begin{array}{lllll}
\delta \boldsymbol{p}^{T} & \boldsymbol{\beta}^{T} & \boldsymbol{C}^{T} & \alpha^{T} & \boldsymbol{\epsilon}^{T}
\end{array}\right]^{T}
$$

where $m_{p}$ is the total number of photodiodes included in the attitude determination system and

$$
\boldsymbol{C}=\left[\begin{array}{lll}
C_{1} & \cdots & C_{m_{p}}
\end{array}\right]^{T}
$$




$$
\begin{aligned}
\alpha & =\left[\begin{array}{lll}
\alpha_{1} & \cdots & \alpha_{m_{p}}
\end{array}\right]^{T} \\
\boldsymbol{\epsilon} & =\left[\begin{array}{lll}
\epsilon_{1} & \cdots & \epsilon_{m_{p}}
\end{array}\right]^{T}
\end{aligned}
$$

The azimuth and elevation of each sensor is expected to remain constant over time (assuming they are not mounted on actuated surfaces). The scale factor may decrease over time due to radiation, but since the degradation is much slower than the frequency of measurements, process noise is sufficient to capture the degradation. The dynamic models of the calibration states are therefore given by Eq. (9), where $\boldsymbol{w}_{C}, \boldsymbol{w}_{\alpha}$, and $\boldsymbol{w}_{\epsilon}$ are each mean zero Gaussian random vectors with covariance matrices $Q_{C}, Q_{\alpha}$, and $Q_{\epsilon}$, respectively.

$$
\begin{gathered}
\dot{\boldsymbol{C}}(t)=w_{C} \\
\dot{\boldsymbol{\alpha}}(t)=\boldsymbol{w}_{\alpha} \\
\dot{\boldsymbol{\epsilon}}(t)=\boldsymbol{w}_{\epsilon}
\end{gathered}
$$

EKFs use partial derivatives of the measurement model [see Eq. (A4)]. The partial derivatives that make up the photodiode portion of the sensitivity matrix $H$ are

$$
\begin{gathered}
\frac{\partial \tilde{I}_{j}}{\partial \delta \boldsymbol{p}}=C_{j} \boldsymbol{n}_{j}^{T}\left[\left(\left.A \boldsymbol{s}\right|_{R}\right) \times\right] \\
\frac{\partial \tilde{I}_{j}}{\partial \boldsymbol{\beta}}=0_{1 \times 3} \\
\frac{\partial \tilde{I}_{j}}{\partial C_{j}}=\left.\boldsymbol{n}_{j}^{T} A \boldsymbol{s}\right|_{R} \\
\frac{\partial \tilde{I}_{j}}{\partial \alpha_{j}}=\left.C_{j}\left[\begin{array}{c}
-\cos \left(\epsilon_{j}\right) \sin \left(\alpha_{j}\right) \\
\cos \left(\epsilon_{j}\right) \cos \left(\alpha_{j}\right) \\
0
\end{array}\right]^{T} A s\right|_{R} \\
\frac{\partial \tilde{I}_{j}}{\partial \epsilon_{j}}=\left.C_{j}\left[\begin{array}{c}
-\sin \left(\epsilon_{j}\right) \cos \left(\alpha_{j}\right) \\
-\sin \left(\epsilon_{j}\right) \sin \left(\alpha_{j}\right) \\
\cos \left(\epsilon_{j}\right)
\end{array}\right]^{T} A \boldsymbol{s}\right|_{R}
\end{gathered}
$$

Equation (10) is derived using the same methods as other vector measurements in the MEKF [29]. These partial derivatives ignore the albedo contribution in Eq. (5), which is a reasonable approximation since the sensitivity due to direct sunlight dominates the sensitivity due to Earth albedo. These equations are used in the MEKF and not USQUE since the UF does not require linearization of the measurement model.

With the new states, measurement model, and partial derivatives in hand, the calibration is integrated into the forms of the MEKF and USQUE shown in the Appendix. The measurement model [Eq. (A7)] and sensitivity matrix of the MEKF [Eq. (A4)] become

$$
\boldsymbol{h}_{k}\left(\hat{\boldsymbol{x}}_{k}^{-}\right)=\left.\left[\begin{array}{c}
\left.A\left(\hat{\boldsymbol{q}}^{-}\right) \boldsymbol{r}_{1}\right|_{R} \\
\vdots \\
\left.A\left(\hat{\boldsymbol{q}}^{-}\right) \boldsymbol{r}_{N}\right|_{R} \\
\left.\left.\hat{C}_{1}^{-} \hat{\boldsymbol{n}}_{1}^{-T}\right|_{B} A\left(\hat{\boldsymbol{q}}^{-}\right) \boldsymbol{s}\right|_{R}+\hat{C}_{1}^{-} \frac{E_{a, 1}\left(\hat{\boldsymbol{n}}_{1}, A\left(\hat{\boldsymbol{q}}^{-}\right)\right)}{E_{\mathrm{AM} 0}} \\
\vdots \\
\left.\left.\hat{C}_{m_{p}}^{-} \hat{\boldsymbol{n}}_{m_{p}}^{-T}\right|_{B} A\left(\hat{\boldsymbol{q}}^{-}\right) \boldsymbol{s}\right|_{R}+\hat{C}_{m_{p}}^{-} \frac{E_{a, m_{p}}\left(\hat{\boldsymbol{n}}_{m_{p}}, A\left(\hat{\boldsymbol{q}}^{-}\right)\right)}{E_{\mathrm{AM} 0}}
\end{array}\right]\right|_{t_{k}}
$$

and

$$
H_{k}\left(\hat{\boldsymbol{x}}_{k}^{-}\right)=\left.\left[\begin{array}{ccccc}
{\left[A(\hat{\boldsymbol{q}}) \boldsymbol{r}_{1} \times\right]} & 0_{3 \times 3} & 0_{3 \times m_{p}} & 0_{3 \times m_{p}} & 0_{3 \times m_{p}} \\
\vdots & \vdots & \vdots & \vdots & \vdots \\
{\left[A(\hat{\boldsymbol{q}}) \boldsymbol{r}_{N} \times\right]} & 0_{3 \times 3} & 0_{3 \times m_{p}} & 0_{3 \times m_{p}} & 0_{3 \times m_{p}} \\
\frac{\partial \tilde{I}_{1}}{\partial \delta \hat{\boldsymbol{p}}} & 0_{1 \times 3} & \frac{\partial \tilde{I}_{1}}{\partial \hat{\boldsymbol{C}}} & \frac{\partial \tilde{I}_{1}}{\partial \hat{\boldsymbol{\alpha}}} & \frac{\partial \tilde{I}_{1}}{\partial \hat{\boldsymbol{\epsilon}}} \\
\vdots & \vdots & \vdots & \vdots & \vdots \\
\frac{\partial \tilde{I}_{m_{p}}}{\partial \delta \hat{\boldsymbol{p}}} & 0_{1 \times 3} & \frac{\partial \tilde{I}_{m_{p}}}{\partial \hat{\boldsymbol{C}}} & \frac{\partial \tilde{I}_{N}}{\partial \hat{\boldsymbol{\alpha}}} & \frac{\partial \tilde{I}_{N}}{\partial \hat{\boldsymbol{\epsilon}}}
\end{array}\right]\right|_{t_{k}}
$$

where the partial derivatives are given by Eqs. (10-14). Only illuminated photodiodes are used in the state update; the rows of $\boldsymbol{h}_{k}$ and $H_{k}\left(\hat{\boldsymbol{x}}_{k}^{-}\right)$that correspond to photodiodes for which the sun is outside the FOV are omitted. Hence, the dimension of $\boldsymbol{h}_{k}$ and $H_{k}\left(\hat{\boldsymbol{x}}_{k}^{-}\right)$ may be different at each update. The logic used to determine if a photodiode is sufficiently illuminated can be based on either the illumination level of the photodiode and/or the estimated attitude and photodiode normal direction combined with the reference sun vector.

From Eq. (9), the state propagation equations of the MEKF are extended to

$$
\begin{aligned}
& \hat{\boldsymbol{C}}_{k+1}^{-}=\hat{\boldsymbol{C}}_{k}^{+} \\
& \hat{\boldsymbol{\alpha}}_{k+1}^{-}=\hat{\boldsymbol{\alpha}}_{k}^{+} \\
& \hat{\boldsymbol{\epsilon}}_{k+1}^{-}=\hat{\boldsymbol{\epsilon}}_{k}^{+}
\end{aligned}
$$

and the equations for state-error covariance propagation are extended to

$$
\begin{gathered}
\Phi=\left[\begin{array}{lll}
\Phi_{11} & \Phi_{12} & \\
\Phi_{21} & \Phi_{22} & \\
& & I_{m_{p} \times m_{p}}
\end{array}\right] \\
G_{k}=\left[\begin{array}{ccc}
-I_{3 \times 3} & 0_{3 \times 3} & \\
0_{3 \times 3} & I_{3 \times 3} & \\
& & I_{3 m_{p} \times 3 m_{p}}
\end{array}\right] \\
Q_{k}=\left[\begin{array}{cccc}
\left(\sigma_{v}^{2} \Delta t+\frac{1}{3} \sigma_{u}^{2} \Delta t^{3}\right) I_{3 \times 3} & \left(\frac{1}{2} \sigma_{u}^{2} \Delta t^{2}\right) I_{3 \times 3} & \\
\left(\frac{1}{2} \sigma_{u}^{2} \Delta t^{2}\right) I_{3 \times 3} & \left(\sigma_{u}^{2} \Delta t\right) I_{3 \times 3} & & \\
& & Q_{C}
\end{array}\right]
\end{gathered}
$$

The UF implementation is accomplished by extending the USQUE in the same manner as the MEKF was extended. There are now $12+$ $6 m_{p}$ sigma points due to the additional calibration states. The sigma points are partitioned for the additional states and propagated as in Eqs. (21) and (22).

$$
\chi_{k}(i) \equiv\left[\begin{array}{c}
\chi_{k}^{\delta p}(i) \\
\chi_{k}^{\beta}(i) \\
\chi_{k}^{C}(i) \\
\chi_{k}^{\alpha}(i) \\
\chi_{k}^{\epsilon}(i)
\end{array}\right], \quad i=0,1,, 12+6 m_{p}
$$




$$
\begin{aligned}
& \chi_{k+1}^{C}(i)=\chi_{k}^{C}(i), \quad i=0,1,12+6 m_{p} \\
& \chi_{k+1}^{\alpha}(i)=\chi_{k}^{\alpha}(i), \quad i=0,1,12+6 m_{p} \\
& \chi_{k+1}^{\epsilon}(i)=\chi_{k}^{\epsilon}(i), \quad i=0,1,12+6 m_{p}
\end{aligned}
$$

The mean observation equations [Eqs. (A34) and (A35)] are extended by appending the photodiode measurement equation as in Eq. (15), and only photodiodes for which the sun is in the FOV are used in the state update. The process covariance matrix is also extended in the same manner as for the MEKF.

With the MEKF and USQUE extended to include the calibration parameters, the process to implement each filter is the same as that used to implement the original filters.

\section{Observability of Photodiode Normal Directions}

The azimuth and elevation angles have been used to parameterize the photodiode normal directions. The azimuth/elevation system used in Eq. (6) and subsequently in Eqs. (13) and (14) is shown in Fig. 2a. It is a common system in which the azimuth is about the $+z$ axis and elevation is measured from the $x-y$ plane. With this system, the azimuth of $\boldsymbol{n}$ when the elevation is \pm 90 deg is undefined and thus unobservable, preventing an accurate estimation of the normal directions of photodiodes mounted parallel to the $z$ axis. To overcome this unobservability, the azimuth/elevation system of Fig. $2 \mathrm{~b}$ is used with photodiodes that are intended to be mounted parallel or near parallel to the $z$ axis. In Fig. $2 \mathrm{~b}$, the azimuth is about the $+y$ axis, and elevation is measured from the $x-z$ plane. This overcomes the observability problem for photodiodes along the $z$ axis and is used only for those photodiodes (it creates the same observability problem for photodiodes along the $y$ axis). In the implementation of the calibration, Eqs. (6), (13), and (14) are rederived to use this system for the $z$ photodiodes.

\section{Application and Results}

Both the UF- and EKF-based approaches to photodiode calibration have been tested through simulation, and their utility has been demonstrated by application to flight data from the RAX-1 and RAX-2 satellites, two CubeSats that include photodiodes for attitude determination. The spacecraft are discussed in Sec. IV.A, and the simulation results are presented in Sec. IV.B. The calibration is applied to flight data in Sec. IV.C, and its impact on sun vector angular accuracy and the resulting attitude determination accuracy is presented in Sec. IV.D.

\section{A. RAX Data}

The calibration filter is applied to data from the Radio Aurora Explorer satellites, RAX-1 and RAX-2. The spacecraft are $3 \mathrm{U}$ CubeSats developed to study space weather irregularities in Earth's ionosphere $[13,32,33]$. RAX-1 launched November 2010 into a $650 \mathrm{~km}, 72$ deg inclination circular orbit, and RAX-2 launched October 2011 into a $102 \mathrm{deg}$ inclination $400 \times 820 \mathrm{~km}$ orbit. The attitude determination sensors are commercial off-the-shelf compo-

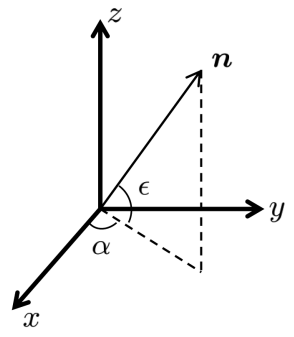

a) Traditional az/el coordinates

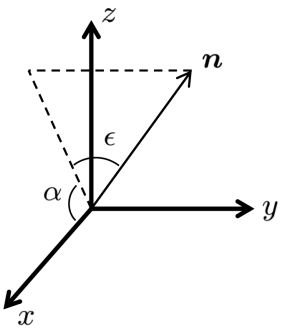

b) Modifie az/el coordinates
Fig. 2 Two different azimuth/elevation (az/el) systems used in the photodiode calibration.
Table 1 The intended azimuth and elevation angles of the photodiode normal directions on RAX-1 (using the azimuth/ elevation system of Fig. 2a); the side panel is the surface to which the sensors are mounted (coordinate system shown in Fig. 3a)

\begin{tabular}{lcccccc}
\hline \hline Photodiode number & 1 & 2 & 3 & 4 & $5-7$ & $8-9$ \\
\hline Side panel & $+x$ & $-x$ & $+y$ & $-y$ & $+z$ & $-z$ \\
$\alpha$, deg & 0 & 180 & 90 & 270 & 0 & 0 \\
$\epsilon$, deg & 0 & 0 & 0 & 0 & 90 & -90 \\
\hline \hline
\end{tabular}

nents and include an Analog Devices ADIS16405 inertial measurement unit (which includes a three-axis gyroscope), a PNI MicroMag3 three-axis magnetometer, and Osram SFH2430 photodiodes. The attitude determination subsystem of RAX-1 is described in [9]. RAX2 uses the same subsystem with the exception of the photodiode configuration: RAX-1 includes photodiodes mounted parallel to the six orthogonal sides of the spacecraft, as indicated by their azimuth and elevation angles given in Table 1 , and RAX-2 includes additional photodiodes mounted at different angles to enable three-component sun sensing for nearly every direction in the body frame. The intended RAX-2 mounting angles, given in Table 2 , were chosen because they provide three-component sensing over nearly the entire body frame while adhering to the CubeSat specifications that limit the height of objects mounted on the satellite surfaces. RAX-2 also includes solar cell coverglass on each photodiode, which was added to reduce radiation-induced degradation of the sensors experienced on RAX-1 [14].

The satellites are pictured in Fig. 3 with their photodiodes circled. The photodiodes on RAX-1 are soldered to the side panels of the spacecraft. On RAX-2, the angled photodiodes are mounted on Delrin wedges, soldered to the solar panels, and staked to the spacecraft. On both satellites, redundant sensors were placed on the $z$ surfaces to avoid shadows from antennas. No high tolerance practices were used in mounting the photodiodes; the wedges were laser cut, and the photodiodes were mounted to the spacecraft by hand. Therefore, it is expected that the photodiode orientations are not well known, particularly for the angled sensors on RAX-2.

Both satellites use a passive magnetic attitude control system, a common low-cost approach to attitude control of nanosatellites. This system uses permanent magnets and magnetic hysteresis material to align the spacecraft to the geomagnetic field and dampen rotational kinetic energy within the first few weeks after deployment from the launch vehicle. The satellites settle to a rotation rate of $1-2 \mathrm{deg} / \mathrm{s}$ about the local magnetic field vector. The time between deployment from the launch vehicle and steady-state dynamics about the local magnetic field vector is typically referred to as tumbling. The RAX-1 and RAX-2 measured angular velocity following deployment into orbit was approximately $20 \mathrm{deg} / \mathrm{s}$. In general, sensor calibration requires sufficient excitation of the sensors of interest. Since RAX spins about the magnetic field vector even when reaching steady-state dynamics, all photodiodes are typically illuminated during a single orbit, and the steady-state dynamics are sufficient for photodiode calibration. Flight data from both the tumbling and steady-state portions of flight are discussed in this section. For actively controlled spacecraft, calibration maneuvers can be performed to achieve sufficient excitation for sensor and actuator calibration.

Flight data used in this section were downloaded from the spacecraft for health assessment and sensor calibration, and the calibration was performed during a ground-based analysis of the flight data. A discussion of real-time calibration and calibration via postprocessing the data is included in Sec. $\underline{\text { V. }}$

\section{B. Simulated Testing}

Before application to flight data, the calibration filters were tested and compared through application to simulated data. The simulated satellite dynamics mimic the flight data: the initial conditions are actual estimated attitude and angular rates from a portion of the flight data, and the only torque included is the control torque provided by permanent magnets, which is the dominant torque. Magnetometer 
Table 2 The intended azimuth and elevation angles of the photodiode normal directions on RAX-2 (using the azimuth/ elevation system of Fig. 2a); the side panel is the surface to which the sensors are mounted (coordinate system shown in Fig. 3b)

\begin{tabular}{|c|c|c|c|c|c|c|c|c|c|c|c|c|c|c|}
\hline Photodiode number & 1 & 2 & 3 & 4 & 5 & 6 & 7 & 8 & 9 & 10 & 11 & 12 & $13-15$ & $16-17$ \\
\hline Side panel & \multicolumn{3}{|c|}{$+x$} & \multicolumn{3}{|c|}{$-x$} & \multicolumn{3}{|c|}{$+y$} & \multicolumn{3}{|c|}{$-y$} & $+z$ & $-z$ \\
\hline$\alpha, \operatorname{deg}$ & 17 & 0 & -17 & -162 & 180 & 162 & 72 & 107 & 90 & -107 & -72 & -90 & 0 & 0 \\
\hline$\epsilon ;$, deg & -10 & 20 & -10 & -10 & 20 & -10 & 10 & 10 & -20 & 10 & 10 & -20 & 90 & -90 \\
\hline
\end{tabular}

and photodiode measurements are simulated by adding zero mean Gaussian noise to simulated body-frame magnetic and sun vector components, and the rate gyroscope measurements are simulated using the model of Eq. (A19).

The sensor noise parameters and initial angular rates used in the simulations are shown in Table 3 . The uncertainty of the photodiode measurement is given in volts because, in practice, voltage rather than current is measured within the photodiode circuit. Voltage is directly proportional to current and is used for the remainder of this paper. The corresponding photodiode angular uncertainty depends on the incidence angle and photodiode scale factor, and the output uncertainty of $0.05 \mathrm{~V}$ corresponds to an angular uncertainty of between 1.0 and $5.7 \mathrm{deg}$ for a scale factor of $3.0 \mathrm{~V}$ (the actual scale factors of the RAX-1 and RAX-2 photodiodes are between 2.5 and $4 \mathrm{~V}$, and a range between 2.5 and $3.5 \mathrm{~V}$ is used in the simulations). The initial angular rates are taken from a portion of the flight data after the postdeployment angular rates have dissipated and the spacecraft dynamics have settled about the geomagnetic field. Compared to the tumbling portion of the flight, these initial conditions are a worst case for sensor excitation, and the simulations demonstrate that this is still sufficient for calibration. The sensor sampling frequency is $1 \mathrm{~Hz}$ for both the gyroscope and attitude sensors. The tuning parameter $\lambda$ of the UF is set to $\lambda=1$ since this value has been demonstrated to provide accurate estimates in previous applications to attitude determination [22,28]. Additionally, like the USQUE, we use $a=1$ and $f=4$. [28].

To compare EKF and UF performance, each filter was applied to 50 trials of simulated data with the initial state estimates set to Gaussian random vectors with mean of the true states and standard deviations given in Table 4. Two simulations of 50 trials each were run to compare the filters under different levels of initial condition accuracy. As seen in Table 4 , simulation 1 uses relatively poor initial estimates of the calibration states, while simulation 2 uses better initial conditions. We will show in Sec. IV.C that the better initial conditions of simulation 2 are more representative of the initial conditions of the flight data. The simulated true values of the photodiode scale factor were set to uniformly distributed random values between 2.5 and $3.5 \mathrm{~V}$, and the simulated true values of the
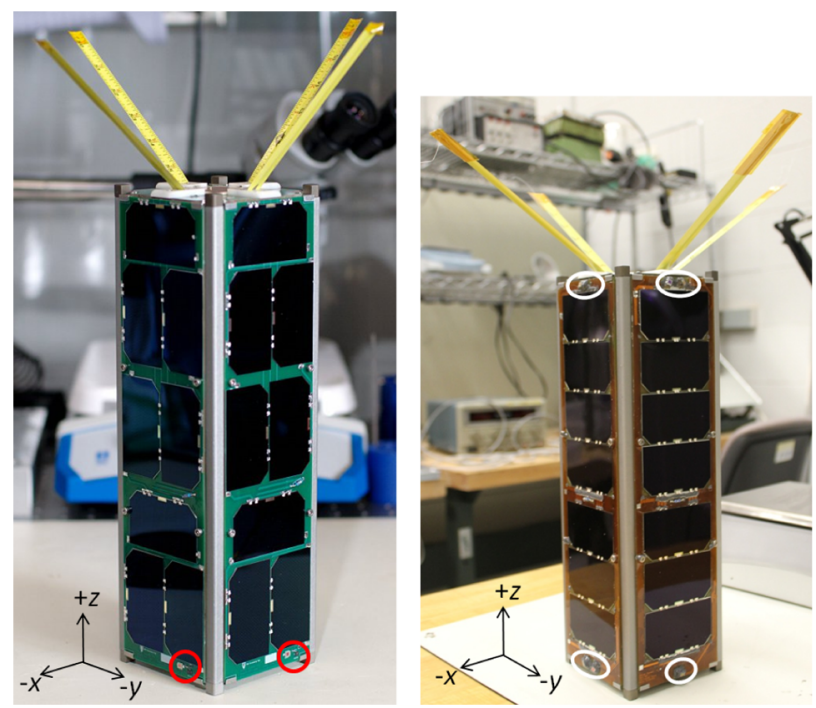

a) RAX-1

b) RAX-2

Fig. 3 The RAX satellites with visible photodiodes circled.
Table 3 Sensor error parameters and initial angular velocity for the simulated data

\begin{tabular}{lc}
\hline \hline Parameter & Value \\
\hline Magnetometer standard deviation & $100 \mathrm{nT}$ \\
Photodiode standard deviation & $0.05 \mathrm{~V}$ \\
Photodiode FOV half-angle & $70 \mathrm{deg}$ \\
Angle random walk $\left(\sigma_{v}\right)$ & $4.89 \times 10^{-4} \mathrm{rad} / \mathrm{s}^{1 / 2}$ \\
Rate random walk $\left(\sigma_{u}\right)$ & $3.14 \times 10^{-5} \mathrm{rad} / \mathrm{s}^{3 / 2}$ \\
Initial angular rate & {$\left[\begin{array}{c}-0.89 \\
0.11 \\
-2.05\end{array}\right] \mathrm{deg} / \mathrm{s}$} \\
\hline
\end{tabular}

Table 4 Initial state estimate accuracy for the simulated testing

\begin{tabular}{lcc}
\hline \hline State & \multicolumn{2}{c}{ Standard Deviation } \\
\cline { 2 - 3 } & Simulation 1 & Simulation 2 \\
\hline$\delta p, \mathrm{deg}$ & 5 & 5 \\
$\beta, \mathrm{deg} / \mathrm{s}$ & 5 & 5 \\
$C, \mathrm{~V}$ & 0.5 & 0.2 \\
$\alpha, \mathrm{deg}$ & 10 & 2 \\
$\epsilon, \mathrm{deg}$ & 10 & 2 \\
\hline \hline
\end{tabular}

photodiode orientation were set the expected states for RAX-2, shown in Table 2. Earth albedo was not included in the simulated testing of the two filters because simply adding the modeled albedo to the simulated measurements and compensating for it within the filter does not affect the relative performance of the two filters. The true accuracy of the albedo model compared to the albedo during the flight data sets is not known, and this is addressed in filter tuning that is discussed in Sec. IV.C.

The error in each state estimate averaged over the 50 trials is shown in Figs. $\underline{4}$ and $\underline{5}$. As seen in Fig. $\underline{4}$, with the initial conditions of simulation 1, the UF provides significantly higher-accuracy state estimates than the EKF. The differences in accuracy are quantified in the caption of each plot. An additional aspect that is not shown in the plots is that the EKF state estimation error falls outside of the stateerror covariance for a significant portion of the states and trials, whereas the UF estimates are within bounds for all states and trials. This shows that, under these initial conditions, the EKF is inconsistent and does not provide reliable state estimates.

As seen in Fig. 5, with the better initial conditions of simulation 2, the difference in state estimation accuracy between the two filters is negligible. Additionally, both the EKF and UF provide state estimates that are consistent with the state-error covariance. Therefore, we conclude that if the initial conditions are sufficiently accurate, the accuracies of the UF- and EKF-based calibrations are the same, but under poor initial conditions, the UF outperforms the EKF. This is consistent with accuracy comparisons between the UF and EKF for various nonlinear estimation problems. Since the initial states of the flight data are unknown, the accuracy of the initial conditions is also unknown, and we apply both the EKF and UF to the flight data. We will show that the initial conditions derived from the measurements are sufficient for use with the EKF.

\section{Application to Flight Data}

Both the UF- and EKF-based methods are applied to estimate the attitude, gyroscope bias, photodiode orientation, and photodiode 


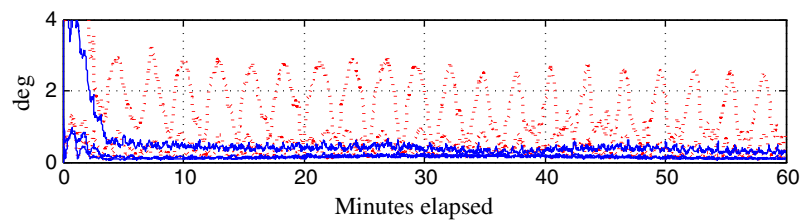

a) Error in the three attitude components. The EKF attitude error exceeds $2 \mathrm{deg}$, while the UF error does not exceed $0.6 \mathrm{deg}$ after convergence

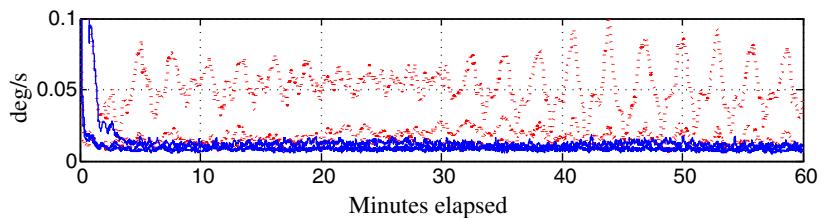

b) Error in the three gyro bias components. The EKF error exceeds $0.05 \mathrm{deg} / \mathrm{s}$, while the UF error does not exceed $0.02 \mathrm{deg} / \mathrm{s}$ after convergence

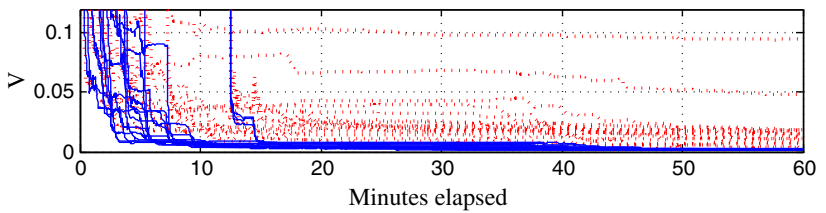

c) Error in the 17 scale factor estimates. The worst-case EKF error is $0.09 \mathrm{~V}$ at the end of the simulation, whereas the worst UF error is below $3 \times 10^{-3} \mathrm{~V}$

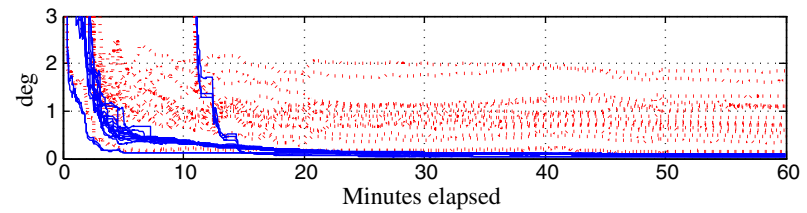

d) Error in the 17 azimuth angle estimates. The worst-case EKF error is $1.8 \mathrm{deg}$ at the end of the simulation, whereas the UF error is below $0.1 \mathrm{deg}$

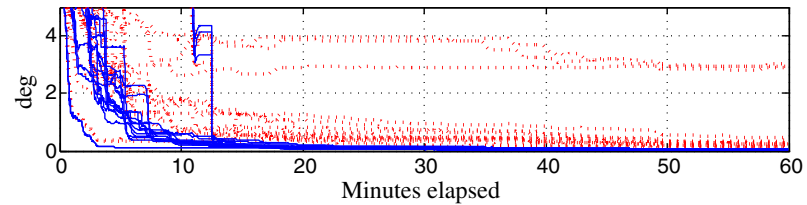

e) Error in the 17 elevation angle estimates. The worst-case EKF error is $3.0 \mathrm{deg}$ at the end of the simulation, wherease the UF error is below $0.1 \mathrm{deg}$

Fig. 4 The average error in each state estimate over the 50 trials with the initial state estimates of simulation 1. In each plot, the UF estimates are shown in solid blue lines, and the EKF estimates are shown in dotted red lines.

scale factors using flight data from RAX-1 and RAX-2. Two data sets from RAX-1 and two data sets from RAX-2 are discussed in this section. Each data set has a $1 \mathrm{~Hz}$ sample frequency and a duration of approximately an orbit, and only the portion of the data during which the spacecraft is in the sun is used with the filters (see Fig. 6). The RAX-1 data sets began 1 December 2010, 08:30:46 UMT, and 30 December 2010, 14:28:15 UMT, and are herein referred to as RAX-1 data sets 1 and 2, respectively. The RAX-2 data sets began 4 November 2011, 18:29:45 UMT, and 12 November 2011, 18:33:00 UMT, and are herein referred to as RAX-2 data sets 1 and 2, respectively. As a sample that is representative of each data set, photodiode measurements from RAX-2 data set 1 are shown in Fig. 6 .

Initial state estimates are required for both estimators. The initial estimate of the scale factor was taken to be the maximum measured output of each photodiode while the spacecraft was tumbling. Recall that this dimensional scale factor is equivalent to the maximum

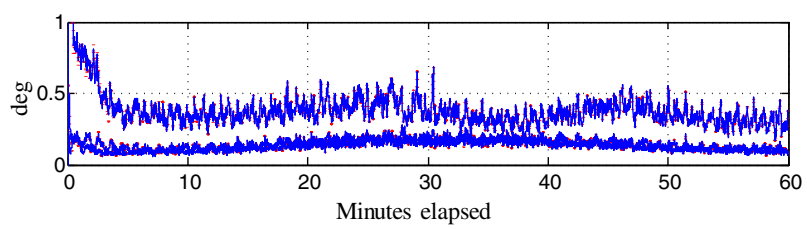

a) Error in the three attitude components

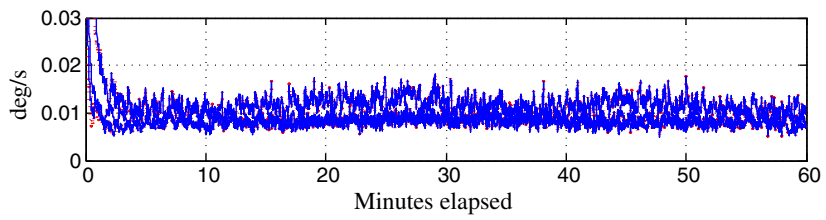

b) Error in the three gyro bias components

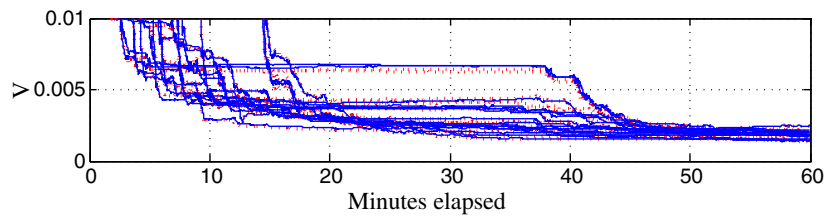

c) Error in the 17 scale factor estimates

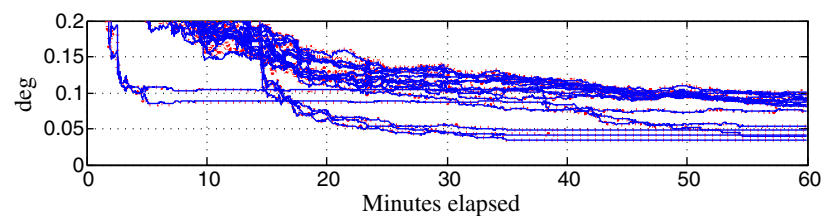

d) Error in the 17 azimuth angle estimates

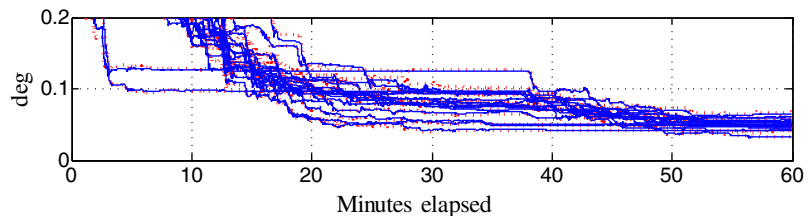

e) Error in the 17 elevation angle estimates

Fig. 5 The average error in each state estimate over the 50 trials with the initial state estimates of simulation 2. In each plot, the UF estimates are shown in solid blue lines, and the EKF estimates are shown in dotted red lines, but the estimates from the two filters are not distinguishable; the difference between the EKF and UF errors is nearly zero.

current output caused by direct sunlight [see Eq. (5)], so the maximum measured output was a reasonable initial estimate. The initial estimate of azimuth and elevation were the intended mounting angles, which were given in Tables 1 and 2 . The initial attitude estimate was calculated from the measured magnetic and sun vectors using the $q$ method [26]. The magnetometers were first calibrated using an on-orbit, attitude-independent method [20], and the sun vector measurement for the initial attitude calculation used the initial calibration parameters. The initial estimate of rate gyroscope bias was zero. The magnetic and sun reference vectors were exported from Analytical Graphics, Inc. (AGI)'s Satellite Toolkit using two-line elements and the SGP4 propagator to estimate the spacecraft position.

Since the filters use the rate gyroscope measurements directly in the attitude propagation, an inherent assumption is that the angular rate is constant between measurements. If the sampling frequency is not sufficient to capture the dynamics, this assumption does not hold, and the process covariance must be increased to account for the uncertainty in the dynamics. The data sets shown in this section are during the tumbling phase of the RAX-1 and RAX-2 missions, and the $1 \mathrm{~Hz}$ gyro sampling frequency is not sufficient to completely capture the dynamics. Thus, the process covariance is increased to rely more heavily on the vector measurements than the gyroscope measurements. This process of adjusting the covariance is referred to as tuning. When working with flight data, filters typically need to be tuned to provide accurate state estimates. The criteria for an accurate 


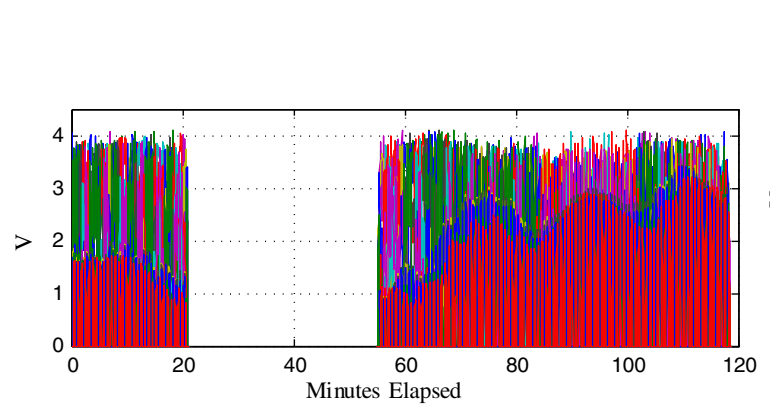

a) Full portion of data

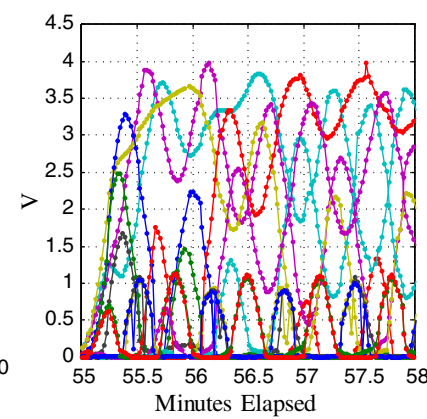

b) Zoomed-in portion

Fig. 6 Raw photodiode measurements (voltage) from RAX-2 data set 1. For this data set, the times of the data used for calibration are 56-118 min.

and near-optimal filter is that the state-error covariance must accurately quantify the state estimation error. During simulated testing, the true states are known (simulated), so the state-error covariance $P$ can be compared directly to the true estimation error. Since the true estimation error is unknown when using flight data and there is no other sensor available as an independent verification of the state estimates, tuning is based on the measurement residuals. The residuals are the differences between the measured vector components and the expected body-frame components calculated by rotating the reference vectors with the estimated attitude. Given the assumption of Gaussian measurement and process noise, the residuals are expected to be zero mean and within the predicted $3 \sigma$ bounds of the combined state error and measurement covariance. Starting with the initial process and measurement covariances, the covariances are then tuned to meet these criteria. The initial process covariance $Q$ is obtained from the assumed rate gyroscope parameters and simulated testing, and the initial measurement covariance is composed of the individual sensor uncertainties. The standard deviation of the PNI magnetometer measurements after on-orbit calibration [20] is approximately 200 and 320 nT for RAX-1 and RAX-2, respectively, and from preflight testing, the approximate standard deviation of the individual photodiode measurements is $0.015 \mathrm{~V}$. To account for uncertainty in the Earth albedo model, an additional uncertainty corresponding to $50 \mathrm{~W} / \mathrm{m}^{2}$ is added to the photodiode measurement uncertainty. This

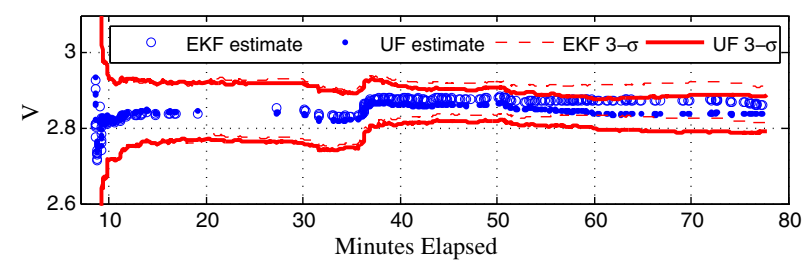

a) $+x$ photodiode

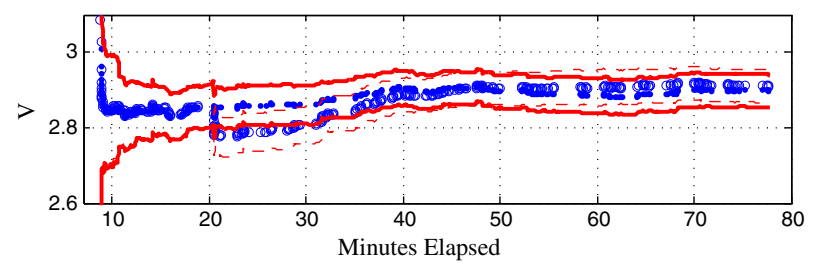

b) $+y$ photodiode

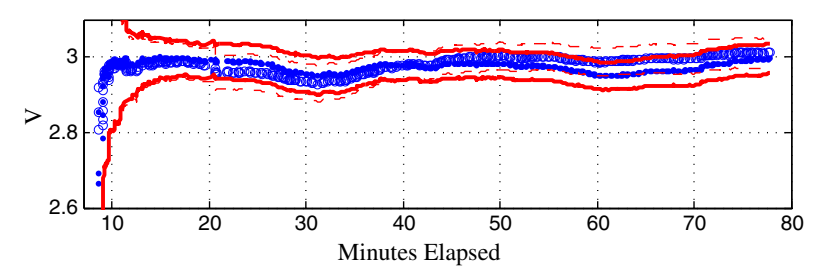

c) $+z$ photodiode

Fig. 7 Scale factor estimates and $\pm 3 \sigma$ bounds for photodiodes from RAX-1 data set 1. uncertainty was found to work well during filter tuning with the flight data. [The average Earth reflectivity is $30.4 \%$, with the variation over Earth's surface shown in Fig. 1a. As seen in Fig. 1b, the standard deviation in reflectivity measurements over 1 year is up to $30 \%$. The albedo (as opposed to reflectivity) is less than this due to the high incident angles of sunlight at the poles. $30 \%$ of $E_{\mathrm{AM} 0}$ is $123 \mathrm{~W} / \mathrm{m}^{2}$, and the impact of albedo also depends on spacecraft attitude. Therefore, the $50 \mathrm{~W} / \mathrm{m}^{2}$ that was found to work well through filter tuning is on the same order of magnitude as the expected uncertainty from variation in Earth reflectivity measurements.] Using the initial estimate of the scale factors, $50 \mathrm{~W} / \mathrm{m}^{2}$ irradiance uncertainty corresponds to a photodiode output of $0.13 \mathrm{~V}$, an order of magnitude greater than the individual photodiode uncertainty.

Plots of the state estimates over time from the tuned filter applied to RAX-1 and RAX-2 data are shown in Figs. 7-12. These show the calibration parameter estimates for three photodiodes in each data set, and the behavior is representative of all the photodiodes on each spacecraft. The state estimates resulting from both the UF and EKF, as well as their $3 \sigma$ bounds from the state-error covariance, are shown. In each plot, the estimate is shown only when a measurement is available for the state update.

In the RAX-1 state estimates (Figs. 7-9), we see that there is a discontinuity in the state estimates at approximately 20 min elapsed. This is due to a pause in the nominal $1 \mathrm{~Hz}$ sampling frequency and gap

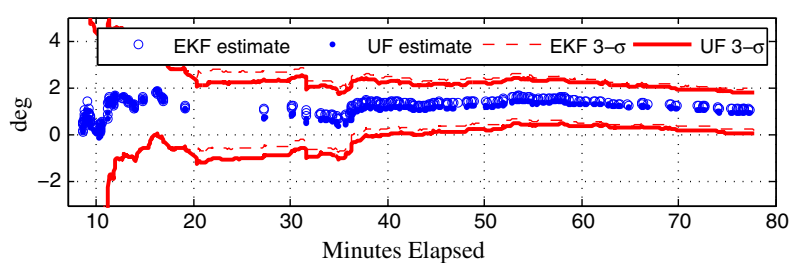

a) $+x$ photodiode

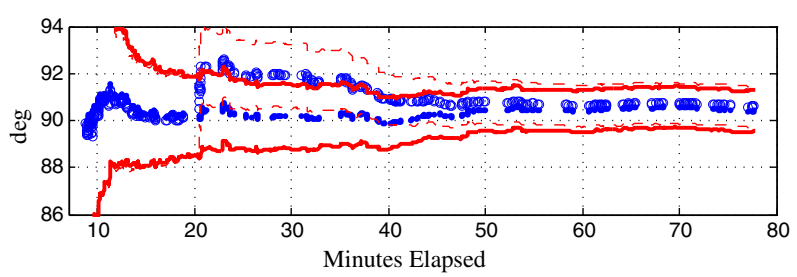

b) $+y$ photodiode

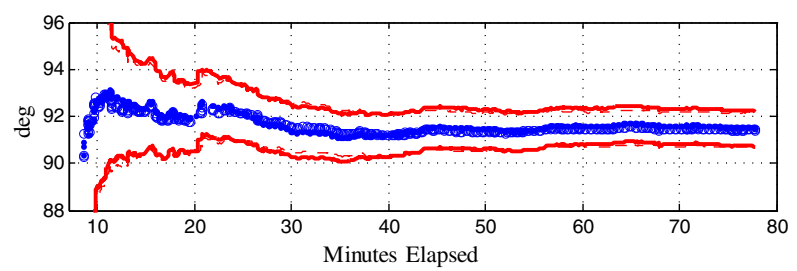

c) $+z$ photodiode

Fig. 8 Azimuth estimates and $\pm 3 \sigma$ bounds for photodiodes from RAX-1 data set 1 . 


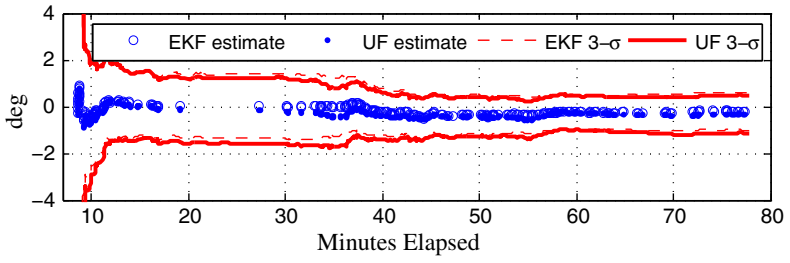

a) $+x$ photodiode

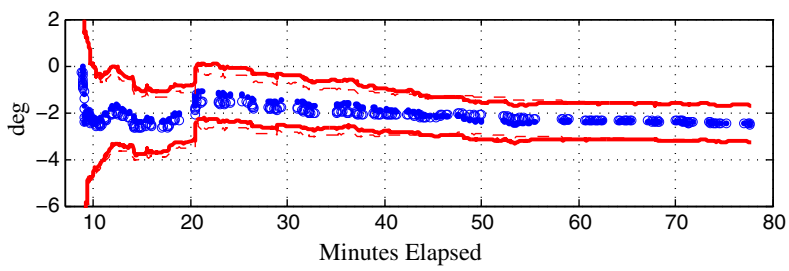

b) $+y$ photodiode

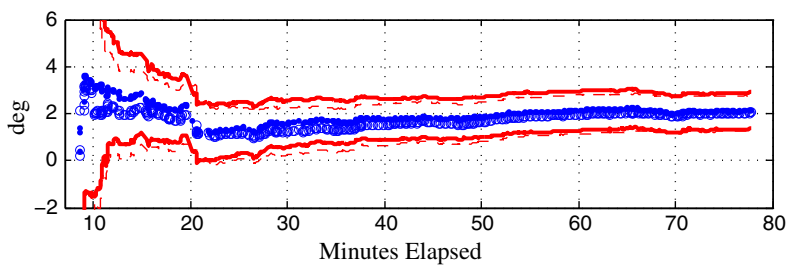

c) $+z$ photodiode

Fig. 9 Elevation estimates and $\pm 3 \sigma$ bounds for photodiodes from RAX1 data set 1.

in the measurements. The EKF takes longer to reconverge than the UF for some states, which is particularly evident in Fig. 8b, but both filters converge to consistent state estimates by 50 min elapsed (20 min after the discontinuity). The scale factor estimates are not exactly constant; oscillations in the estimates even after convergence are due to the mismatch between Earth albedo and the model. (The deviations from a constant estimate could also be due to temperature dependence, but from a visual comparison of the measurements of nearby temperature sensors to the state estimates, consistent correlation between the scale factor estimate and temperature was not evident. A thorough characterization of the temperature dependence of these photodiodes was not preformed preflight, and this is an area that could be addressed in future work.) From the plots, we see that when the filters are converged the differences in the state estimates of the EKF and UF are negligible.

In the RAX-2 state estimates (Figs. 10-12), we see that the estimates converge to a near-constant value at approximately $80 \mathrm{~min}$ elapsed time (20 min into the sun-illuminated portion of the data). Like the RAX-1 estimates, the differences between the EKF- and UFbased state estimates is negligible, and a slightly faster convergence time is evident in the UF estimates.

In each plot, we see that the estimates converge to consistent values. This as well as the residuals criteria discussed previously indicate that the filter is providing accurate state estimates. We use two metrics as an additional verification of the accuracy of the state estimates. First, comparing the estimates of the azimuth and elevation from the different data sets from each satellite, which we expect to be constant over time, the estimated $3 \sigma$ bounds overlap, which demonstrates consistency in the estimates. Additionally, the effectiveness of the calibration can be seen in comparing the measured sun vector magnitude using the initial and final calibration parameters, which is an attitude-independent verification metric. The magnitude of the measured sun vector should be 1 . Histograms of this vector magnitude - from the RAX-2 data sets, which were calculated with the

\footnotetext{
This pause is specific to the RAX flight data and is an artifact of the flight software. The pause in measurements is when the spacecraft is writing the data to flash memory.

**The vector magnitude does not have a Gaussian distribution. The sum of the squares of components with Gaussian distributions has a $\chi^{2}$ distribution.
}

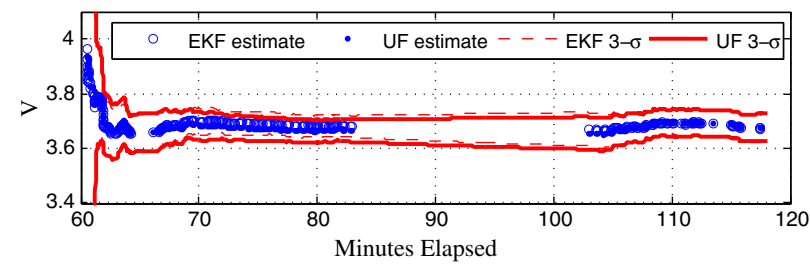

a) Photodiode \#1

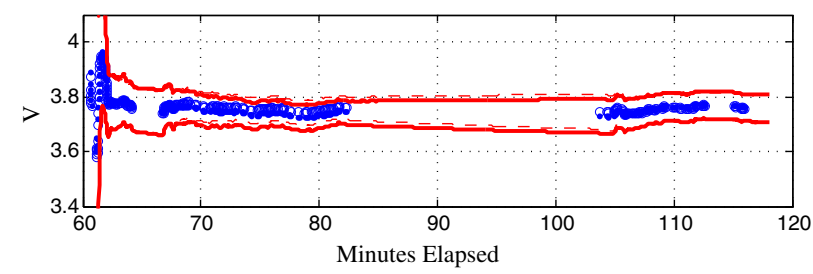

b) Photodiode \#2

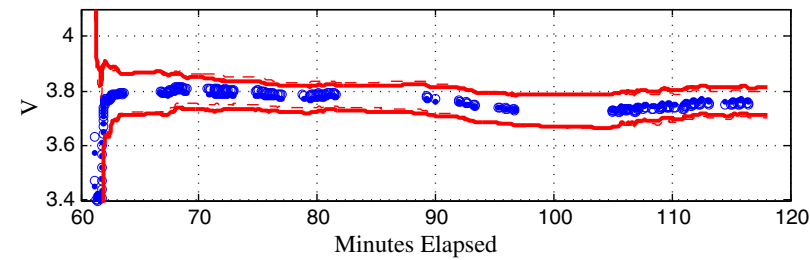

c) Photodiode \#3

Fig. 10 Scale factor estimates and $\pm 3 \sigma$ bounds for photodiodes from RAX-2 data set 1.

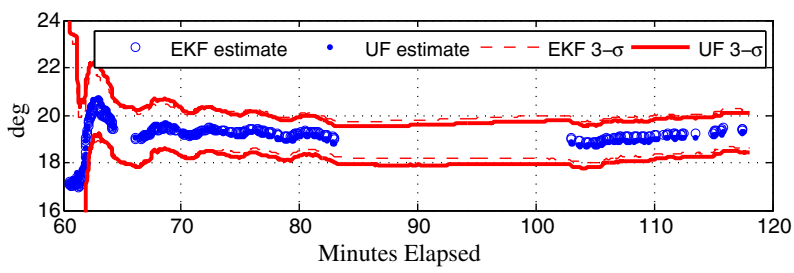

a) Photodiode \#1

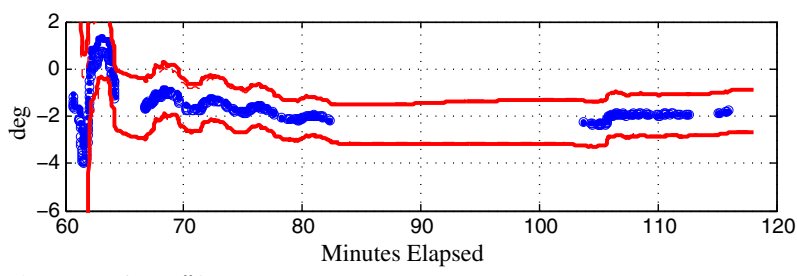

b) Photodiode \#2

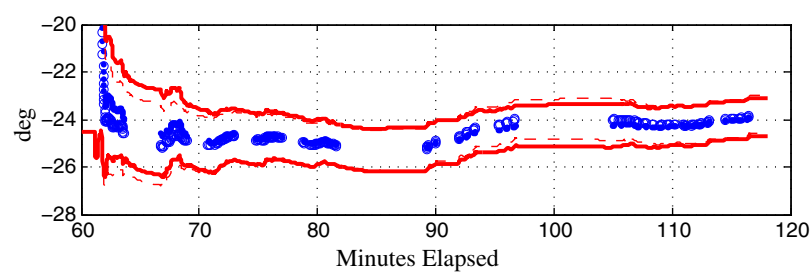

c) Photodiode \#3

Fig. 11 Azimuth estimates and $\pm 3 \sigma$ bounds for photodiodes from RAX2 data set 1.

albedo-corrected photodiode measurements and both the initial and calibrated photodiode parameters, are shown in Figs. 13 and 14 . (RAX-2 data are used to show the attitude-independent verification metrics because a three-component sun vector is required for the attitude-independent metrics, and with the angled photodiodes on RAX-2, sun vectors can be calculated for a much higher percentage of data than RAX-1. But the improvement in vector magnitude 


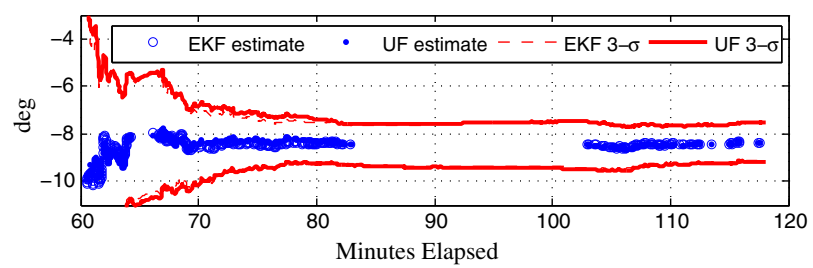

a) Photodiode \#1

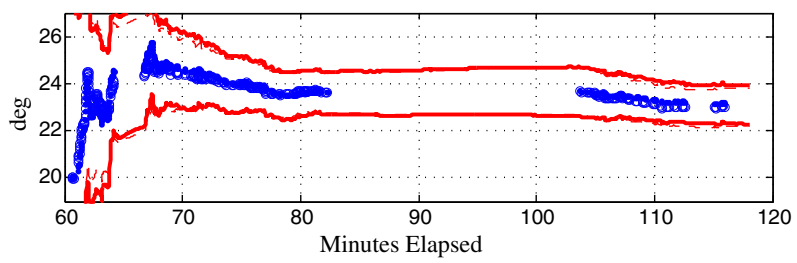

b) Photodiode \#2

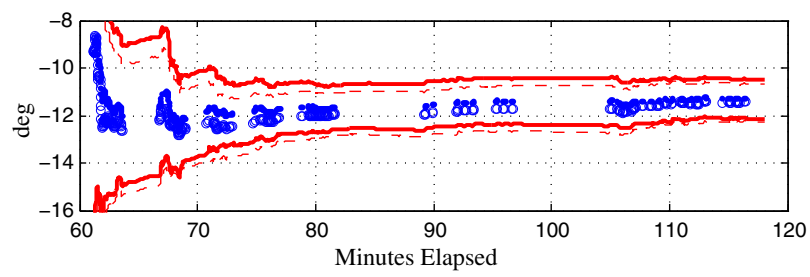

c) Photodiode \#3

Fig. 12 Elevation estimates and $\pm 3 \sigma$ bounds for photodiodes from RAX-2 data set 1.

is evident in all data sets). In the first data set, the mean vector magnitude improves from 0.925 to 0.993 , and the standard deviation decreases from 0.029 to 0.022 when using the on-orbit-estimated parameters rather than initial parameters. In the second data set, the mean improves from 0.932 to 0.999 , and the standard deviation decreases from 0.032 to 0.021 .

The importance of including Earth albedo in the calibration is evident by comparing the magnitude of the measured sun vector calculated with the raw photodiode measurements to the magnitude calculated with albedo-compensated measurements. This is shown in Fig. 15 for the RAX-2 data sets. There are clear deterministic trends in the magnitude calculated from the uncompensated measurements that have been removed by subtracting the modeled albedo contribution from the measurements. Since the albedo contribution is attitude dependent, this demonstrates the need for simultaneous attitude estimation and calibration as opposed to an attitude-independent calibration. These trends were also seen in an initial attitude-independent attempt at calibrating the RAX-1 photodiodes [14], which required three simultaneously illuminated sensors and $\overline{\mathrm{did}}$ not account for Earth albedo. The new recursive method presented in this paper overcomes both the adverse effect of albedo and the need for multiple illuminated sensors in the calibration.
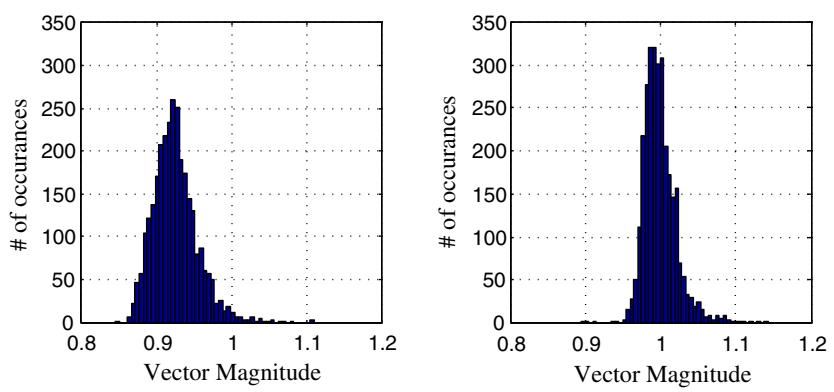

Fig. 13 Histograms of the magnitude of the measured sun vector from RAX-1 data set 1 using the initial (left) and calibrated (right) sensor parameters.
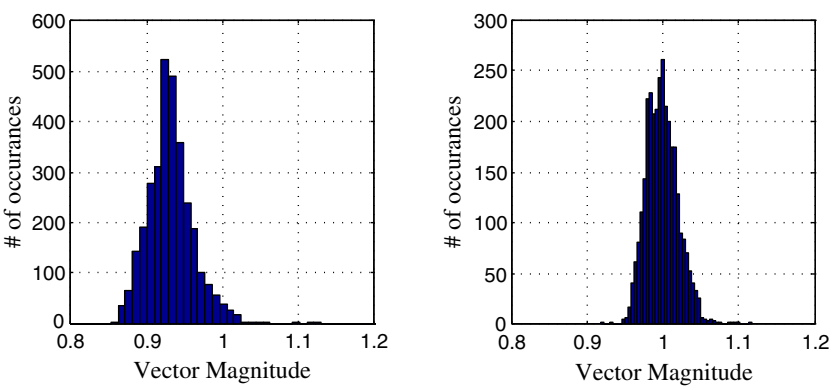

Fig. 14 Histograms of the magnitude of the measured sun vector from RAX-2 data set 2 using the initial (left) and calibrated (right) sensor parameters.

\section{Impact on Sun Vector Angular Accuracy and Attitude Determination}

The difference between the initial calibration parameters, which were the intended mounting angles and the maximum measured output of each photodiode while the spacecraft was tumbling, and the estimates from on-orbit calibration is significant. In the RAX-2 data, the improvement in the elevation and azimuth estimates ranged from 0-9 deg, and the improvement in scale factor ranged from 0.17 to $0.46 \mathrm{~V}$, which is equivalent to $4.4-12.7 \%$ of the calibrated scale factor. For RAX-1, the azimuth and elevation improvement ranged from 0 to $4 \mathrm{deg}$, and the improvement in scale factor ranged from 0.09 to $0.29 \mathrm{~V}$, which is equivalent to $3.4-10.2 \%$ of the calibrated scale factor. The scale factor estimates from the RAX-1 data sets are shown in Table 5. (There are nine photodiodes on RAX-1, but only five are shown in the table. This is because measurements from the redundant photodiodes on the $z$ surfaces were not downloaded for analysis. Additionally, the $-z$ photodiode is shadowed for a significant portion of the measurements and is also affected by reflections from the antenna when it is not shadowed, and therefore its measurements were not included in the calibration). A decrease in the photodiode scale factor as the satellite spends more time on orbit is evident. This is hypothesized to be due to UV radiation. Photodiodes are known to degraded due to UV radiation, and no protective measures were taken to prevent this on RAX-1. This highlights the utility of the calibration in tracking sensor parameters on orbit. On RAX-2, a solar cell coverglass was applied to the photodiodes, and the degradation of RAX-1 is not evident.

Histograms of angular differences between the measured sun vector using the initial and calibrated parameters for the RAX-2 data sets are shown in Fig. 16. The mean improvements in the sun vector angular accuracies are 9.1 and $10.2 \mathrm{deg}$ from the two data sets, which is quite significant given that the angular accuracy of photodiodes is on the order of degrees. The poor alignment of the photodiodes is not surprising given the manual process used to integrate them to the spacecraft. The mean improvement in the sun vector angular accuracy for the RAX-1 data sets is 5.6 and $6.4 \mathrm{deg}$. In application to other spacecraft, the degree of improvement resulting from the on-orbit calibration will be dependent on the quality of the preflight calibration parameters.

The $1 \sigma$ attitude estimation accuracies during the simultaneous attitude estimation and photodiode calibration from RAX-1 data set 1 and RAX-2 data set 1 are shown in Fig. 17. The three components are the uncertainty in rotation about the $x, y$, and $z$ axes of the body-fixed frame. We see that the accuracy of the three components is better than 2 deg $1 \sigma$ and 1 deg $1 \sigma$ for RAX-1 and RAX-2, respectively, for most of the duration that the spacecraft is in the sun. The higher uncertainty between 90 and 100 min for RAX-2 is caused by the relative alignment of the sun and magnetic vectors in the body frame. During this time period, the vectors are nearly parallel, significantly reducing the amount of information available for attitude determination. This condition does not arise during the RAX-1 data set (the spacecraft are in different orbits). Both of these data sets are taken during the tumbling portion of the flight, but the angular velocities of RAX-1 and RAX-2 during these data sets is nearly the same, and the same process covariance is used for each data set. The cause of the better 


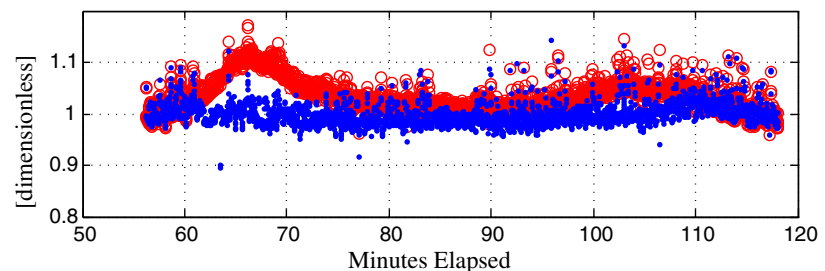

a) Data set 1

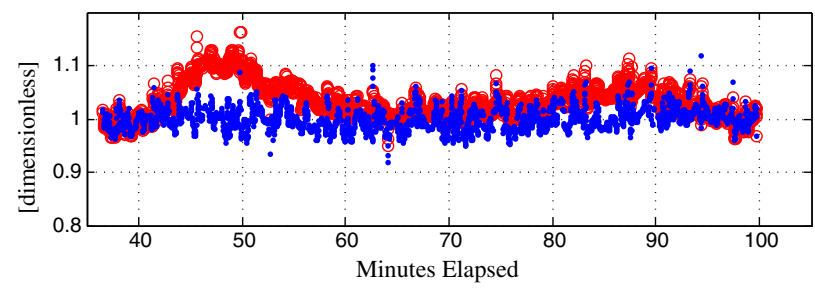

b) Data set 2

Fig. 15 Magnitude of the measured sun vector over time for the two RAX-2 data sets using both the uncompensated photodiode measurements (red circles) and the albedo-compensated measurements (blue dots). These magnitudes were calculated with the estimated calibration parameters. The albedocompensated series of data is the same data as the calibrated histograms of Figs. 13 and 14.

attitude accuracy of RAX-2 compared to RAX-1 is the additional photodiodes in the attitude determination system.

In the data sets used so far, the $1 \mathrm{~Hz}$ gyro sampling frequency was not sufficient to capture the spacecraft dynamics, and the process covariance matrix was inflated to account for the dynamics uncertainty as discussed in Sec. IV.C. Higher-accuracy attitude estimation was achieved when the gyro measurements sufficiently capture the dynamics, and the process covariance did not have to be increased significantly beyond that of Eqs. (A18) and (A23). This is demonstrated by Fig. 18, which shows the attitude accuracy during a data set from later in the RAX-2 mission (9 December 2011) after the passive magnetic control system had dampened the rotational kinetic energy and aligned the spacecraft $z$ axis with Earth's magnetic field. We see that the $1 \sigma$ attitude accuracy in the $x$ and $y$ axes was better than $0.5 \mathrm{deg}$ when the spacecraft was in the sun. The $z$ axis (spin axis) had the least accuracy because the spacecraft was spinning about the magnetic vector, which minimized the amount of nonredundant information that the gyroscope and magnetometer provided. The decrease in accuracy of all three components at $72-105$ min was when the spacecraft entered eclipse and the photodiode measurements were no longer available. This plot demonstrates the full potential of the lowcost, commercial off-the-shelf attitude sensors used in conjunction with the on-orbit calibration methods of this paper.

\section{Discussion and Future Work}

We have used a three-axis rate gyroscope and three-axis magnetometer in addition to the photodiodes for the photodiode calibration. Since the calibration is a recursive method, either a rate gyroscope or a dynamic model is required for fusion with the vector measurements. The use of a rate gyroscope instead of a dynamic model has become a common approach for attitude estimation [25], but the use of a dynamic model with no rate gyroscope has also been demonstrated $[34,35]$ and can be used for photodiode calibration with the formulation presented in Sec. III. The calibration can also be accomplished without a magnetometer or other vector sensor, but the state estimation accuracy will be worse than with the additional attitude sensor since the sun sensors alone provide only two-axis information (the spin about the sun vector is the third axis). We included the magnetometer since it is available and results in significantly better accuracy compared to that using only the photodiodes. We have done initial testing of the calibration filter with only the gyroscope and photodiode measurements and have demonstrated convergence, but a thorough study on the accuracy when using photodiodes as the only vector measurement is left for future work.

Application of the EKF- and UF-based calibration to both simulated and flight data showed that the UF is required when the initial state estimates are relatively poor, but the accuracy of the EKF matches that of the UF if the initial state estimates are sufficiently accurate. The initial state estimates used with the flight data, which

Table 5 Scale factor estimates from the RAX-1 data sets

\begin{tabular}{lccccc}
\hline \hline Photodiode number & 1 & 2 & 3 & 4 & 5 \\
\hline Maximum measurement, V & 3.02 & 3.12 & 3.00 & 2.98 & 3.15 \\
Data set 1 estimate, V & 2.84 & 2.95 & 2.90 & 2.89 & 3.00 \\
Data set 2 estimate, V & 2.76 & 2.92 & 2.81 & 2.79 & 2.86 \\
\hline \hline
\end{tabular}

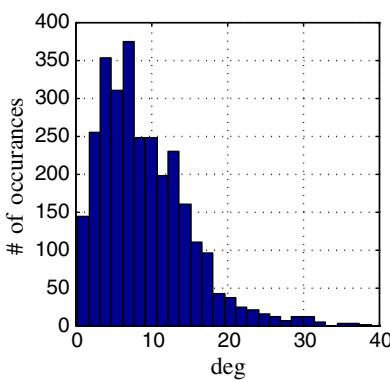

a) RAX-2 data set 1

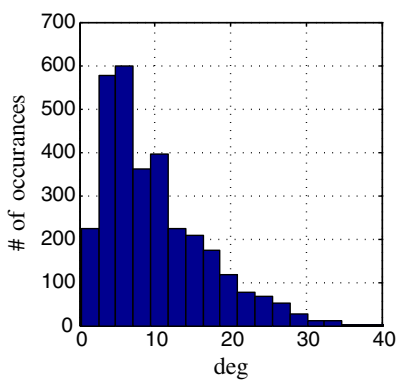

b) RAX-2 data set 2
Fig. 16 Histograms of the angular difference between the measured sun vector using the initial and calibrated photodiode parameters from the RAX-2 data sets. The mean from data set 1 is $9.1 \mathrm{deg}$, and the mean from data set 2 is 10.2 deg.

for the attitude and photodiode scale factors were derived from the measurements and for the photodiode orientation were assumed to be the designed values, were sufficiently accurate for use with the EKF. Therefore, it is reasonable to assume that the EKF is sufficient for photodiode calibration with other spacecraft of comparable or better specifications. This would facilitate real-time implementation, whereas the UF is not well suited for real-time implementation on small spacecraft without significant parallel-processing capability. In general, if the processing power is available to facilitate the UF, such as during a ground-based analysis, the UF should be used over the EKF since its performance is better over a broader range of initial conditions.

In the application of this calibration method to RAX, the flight data are downloaded from the spacecraft and processed on the ground. Real-time attitude estimation is not required on RAX, and downloading batches of data periodically throughout the mission is part of normal RAX operations. Nonetheless, extended Kalman filters are well suited for real-time implementation. Murrell's version of the EKF can be used to reduce the size of matrices required for inversion to $3 \times 3$, and discrete attitude propagation can be used to reduce the computational requirements [36]. The only aspect of the EKF-based calibration method that is not well suited for online implementation in its current form is the Earth albedo model. For online implementation, the albedo model could be simplified [37] or precalculated and tabulated onboard. Investigation into these options is left for future work. Offline calibration can be advantageous since it allows for a thorough inspection of the measurement residuals and tuning parameters, and real-time sensor correction can be accomplished even with offline calibration by uploading the calibration parameters to the spacecraft.

Two other areas for further investigation include the degradation in state estimation accuracy as the number of photodiodes increases and the use of a colored noise model. The maximum number of photodiodes used for the calibration was 17 , dictated by the number of photodiodes used on RAX-2. It is reasonable to assume that increasing the number of photodiodes will begin to degrade the accuracy of the state estimates due to information dilution. Additionally, given that the uncertainty in Earth albedo varies over Earth's surface (see Fig. 1b), a colored noise model or a Gaussian noise model with 


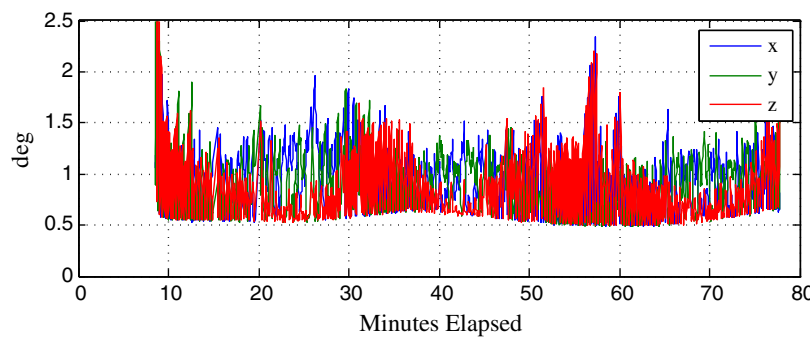

a) RAX-1 data set 1

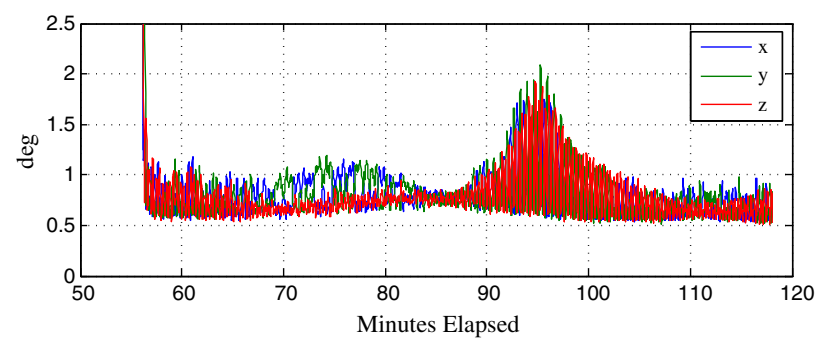

b) RAX-2 data set 1

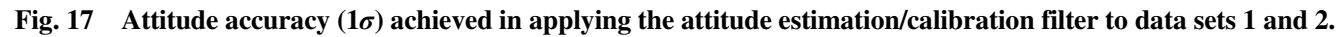

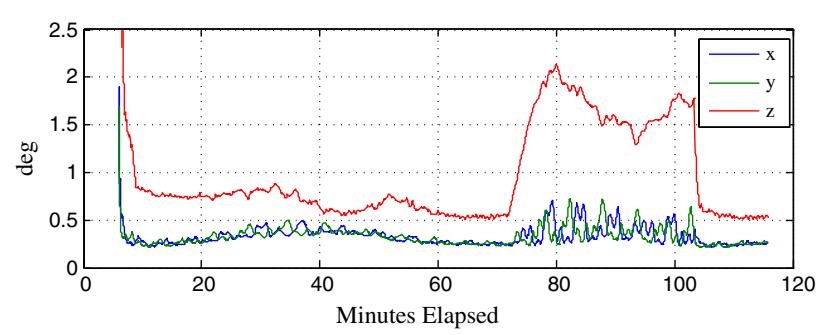

Fig. 18 Attitude accuracy of a third RAX-2 data set, which was collected 9 December 2011, 16:00:00 UMT.

time-varying covariance may provide improved results instead of the Gaussian noise model of constant covariance used in this work. This was not pursued since the current implementation already provides a significant improvement in the sun vector estimation compared to the use of preflight calibration. Both of these areas of study are left for future work.

In addition to attitude determination, the photodiode calibration can be used to track the orientation of actuated surfaces on a spacecraft. For example, if photodiodes are placed on actuated solar panels, then the filter presented in this paper can be used to estimate the orientation of the actuated panels relative to the body-mounted attitude sensors.

\section{Conclusions}

A method for on-orbit photodiode calibration has been developed to estimate the orientations and scale factors of photodiodes in an attitude determination system. The calibration has been formulated for use with either an extended Kalman filter or an unscented filter to simultaneously estimate the spacecraft attitude and the calibration parameters, and it can be applied to any number of photodiodes in an arbitrary configuration on the spacecraft. The importance of the attitude-dependent approach and the use of an Earth albedo model was demonstrated. In application to RAX-2, which uses photodiodes, magnetometers, and a three-axis rate gyroscope for attitude determination, the calibration improved the accuracy of the measured sun vector by an average of $10 \mathrm{deg}$. This calibration enabled the most accurate performance of the attitude determination system with the given hardware. With the combination of calibrated photodiodes as well as a low-cost magnetometer and gyroscope, attitude accuracies of better than 1 deg $1 \sigma$ have been demonstrated.

\section{Appendix A: MEKF and USQUE Overview}

In this Appendix, we provide the equations used on the MEKF and USQUE. A complete derivation and explanation of the MEKF and USQUE are beyond the scope of this paper, but we provide an overview of each while assuming the reader is familiar with Kalman filtering, unscented filtering, and spacecraft attitude estimation [2529,36]. The equations for both attitude estimation filters are given here with sufficient detail for the reader to implement them, and this provides the background information that is used in extending both estimators for photodiode calibration.

Both EKFs and UFs can be used to estimate the states of the system with the general form given by Eq. (A1), where $x_{k}$ is the state of the system at time $k, \tilde{\boldsymbol{y}}_{k}$ is the measurement vector at time $k, \boldsymbol{f}()$ and $\boldsymbol{h}()$ are nonlinear functions, $G_{k}$ is a gain, and $\boldsymbol{w}_{k} \sim N\left(0, Q_{k}\right)$ and $\nu_{k} \sim$ $N\left(0, R_{k}\right)$ are random vectors that quantify model and measurement uncertainty. $Q_{k}$ and $R_{k}$ are referred to as the process covariance and measurement covariance, respectively,

$$
\begin{gathered}
\boldsymbol{x}_{k+1}=\boldsymbol{f}\left(\boldsymbol{x}_{k}, k\right)+G_{k} \boldsymbol{w}_{k} \\
\tilde{\boldsymbol{y}}_{k}=\boldsymbol{h}\left(\boldsymbol{x}_{k}, k\right)+\boldsymbol{\nu}_{k}
\end{gathered}
$$

\section{A1 MEKF}

The MEKF is a widely used approach to spacecraft attitude estimation for vehicles with a three-axis rate gyroscope and at least one attitude sensor $[25,29,36]$. The filter estimates six states: a $3 \times 1$ attitude error vector $\delta \bar{p}$ and the $3 \times 1$ rate gyroscope bias $\boldsymbol{\beta}$. The attitude error vector, which parameterizes error quaternions $\delta \boldsymbol{q}=$ [ $\delta \boldsymbol{\rho} \quad \delta q_{4}$ ], is combined with quaternions being propagated within the filter to estimate spacecraft attitude. Various three-component attitude error vectors can be used, such as the Gibb vector, and each results in the same approximate relationship to the error quaternion, given in Eq. (A2), that is valid to second order (sufficient for the EKF) [29],

$$
\delta \boldsymbol{q}(\delta \boldsymbol{p}) \approx\left[\begin{array}{c}
\delta \boldsymbol{p} / 2 \\
1-\delta \boldsymbol{p}^{2} / 8
\end{array}\right]
$$

The state vector is given by $\boldsymbol{x}=\left[\begin{array}{ll}\delta \boldsymbol{p}^{T} & \boldsymbol{\beta}^{T}\end{array}\right]^{T}$. Unlike the general form of an EKF, which combines a dynamic model with sensor measurements, the MEKF does not use a dynamic model. Instead, the bias-corrected gyroscope measurements are used directly to propagate the attitude kinematics, which alleviates the difficulties of accurately modeling spacecraft dynamics [25].

The following procedure is used for attitude estimation with the MEKF. First, the state vector estimate is initialized to $\hat{\boldsymbol{x}}_{0}=$ $\left[\begin{array}{ll}0_{1 \times 3} & \hat{\boldsymbol{\beta}}_{0}^{T}\end{array}\right]^{T}$, the state-error covariance is initialized to $P_{0}$, and the quaternion estimate is initialized to $\hat{\boldsymbol{q}}_{0}$. The attitude error vector is initialized to zero because the attitude is quantified completely by the quaternion estimate. Then, the Kalman gain is computed using Eqs. (A3) and (A4). Throughout the equations, the superscripts - and + denote quantities before and after the state update, respectively. In Eq. (A4), $A(\boldsymbol{q})$ is the attitude matrix corresponding to the quaternion $\boldsymbol{q}, \boldsymbol{r}_{i}$ is the $i$ th vector measurement, and $\times$ denotes the skew-symmetric cross-product matrix. The state estimate and error covariance are updated via the conventional EKF update of Eqs. (A5A7). After the state update, the attitude error portion of the updated state $\delta \hat{\boldsymbol{p}}_{k}^{+}$is used to update the quaternion estimate using Eq. (A8). Equation (A8) is from where the term multiplicative comes: the attitude error that was updated via the traditional additive update was passed to the quaternion estimate using quaternion multiplication. Next, the bias estimate is used to correct the rate gyro measurement as in Eq. (A9), and the attitude estimate is then propagated using Eqs. (A $\overline{10}-\mathrm{A} 12)$. Since the attitude error has been passed to the quaternion estimate, the attitude error estimate is reset to zero as in Eq. (A13). The bias estimate remains constant until the next time step as in Eq. (A14). Finally, the state-error covariance is propagated using 
Eqs. (A15-A18), where $\Delta t$ is the time increment until the next available measurement. In Eq. (A18), $\sigma_{u}$ and $\sigma_{v}$ are characteristics of the rate gyroscope, discussed in the next paragraph. After propagation to the next time step, the process is repeated with the next available measurement starting with the Kalman gain computation of Eq. (A3),

$$
\begin{aligned}
& K_{k}=P_{k}^{-} H_{k}^{T}\left(\hat{\boldsymbol{x}}_{k}^{-}\right)\left[H_{k}\left(\hat{\boldsymbol{x}}_{k}^{-}\right) P_{k}^{-} H_{k}^{T}\left(\hat{\boldsymbol{x}}_{k}^{-}\right)+R_{k}\right]^{-1} \\
& \left.H_{k}\left(\hat{\boldsymbol{x}}_{k}^{-}\right) \equiv \frac{\partial \boldsymbol{h}}{\partial \boldsymbol{x}}\right|_{\hat{x}_{k}^{-}}=\left.\left[\begin{array}{cc}
{\left[A\left(\hat{\boldsymbol{q}}^{-}\right) \boldsymbol{r}_{1} \times\right]} & 0_{3 \times 3} \\
\vdots & \vdots \\
{\left[A\left(\hat{\boldsymbol{q}}^{-}\right) \boldsymbol{r}_{N} \times\right]} & 0_{3 \times 3}
\end{array}\right]\right|_{t_{k}} \\
& P_{k}^{+}=\left[I-K_{k} H_{k}\left(\hat{\boldsymbol{x}}_{k}^{-}\right)\right] P_{k}^{-} \\
& \hat{\boldsymbol{x}}_{k}^{+}=\hat{\boldsymbol{x}}_{k}^{-}+K_{k}\left[\tilde{\boldsymbol{y}}_{k}-\boldsymbol{h}\left(\hat{\boldsymbol{x}}_{k}^{-}\right)\right] \\
& \boldsymbol{h}\left(\hat{\boldsymbol{x}}_{k}^{-}\right)=\left.\left[\begin{array}{c}
A\left(\hat{\boldsymbol{q}}^{-}\right) \boldsymbol{r}_{1} \\
\vdots \\
A\left(\hat{\boldsymbol{q}}^{-}\right) \boldsymbol{r}_{N}
\end{array}\right]\right|_{t_{k}} \\
& \hat{\boldsymbol{q}}_{k}^{+}=\hat{\boldsymbol{q}}_{k}^{-}+\frac{1}{2} \Xi\left(\hat{\boldsymbol{q}}_{k}^{-}\right) \delta \hat{\boldsymbol{p}}_{k}^{+} \\
& \hat{\boldsymbol{\omega}}_{k}^{+}=\tilde{\boldsymbol{\omega}}_{k}-\hat{\boldsymbol{\beta}}_{k}^{+} \\
& \hat{\boldsymbol{q}}_{k+1}^{-}=\Omega\left(\hat{\boldsymbol{\omega}}_{k}^{+}\right) \hat{\boldsymbol{q}}_{k}^{+}
\end{aligned}
$$$$
\Omega\left(\hat{\boldsymbol{\omega}}_{k}^{+}\right) \equiv\left[\begin{array}{cc}
\cos \left(0.5\left\|\hat{\boldsymbol{\omega}}_{k}^{+}\right\| \Delta t\right) I_{3 \times 3}-\left[\hat{\psi}_{k}^{+} \times\right] & \hat{\boldsymbol{\psi}}_{k}^{+} \\
-\hat{\psi}_{k}^{+T} & \cos \left(0.5\left\|\hat{\boldsymbol{\omega}}_{k}^{+}\right\| \Delta t\right)
\end{array}\right]
$$$$
\hat{\psi}_{k}^{+} \equiv \sin \left(0.5\left\|\hat{\boldsymbol{\omega}}_{k}^{+}\right\| \Delta t\right) \hat{\boldsymbol{\omega}}_{k}^{+} /\left\|\hat{\boldsymbol{\omega}}_{k}^{+}\right\|
$$$$
\delta \hat{\boldsymbol{p}}_{k+1}^{-}=0_{3 \times 1}
$$$$
\hat{\boldsymbol{\beta}}_{k+1}^{-}=\hat{\boldsymbol{\beta}}_{k}^{+}
$$$$
P_{k+1}^{-}=\Phi_{k} P_{k}^{+} \Phi_{k}^{T}+G_{k} Q_{k} G_{k}^{T}
$$$$
\Phi=\left[\begin{array}{ll}
\Phi_{11} & \Phi_{12} \\
\Phi_{21} & \Phi_{22}
\end{array}\right]
$$

$$
\Phi_{11}=I_{3 \times 3}-[\hat{\boldsymbol{\omega}} \times] \frac{\sin (\|\hat{\boldsymbol{\omega}}\| \Delta t)}{\|\hat{\boldsymbol{\omega}}\|}+[\hat{\boldsymbol{\omega}} \times]^{2} \frac{\{1-\cos (\|\hat{\boldsymbol{\omega}}\| \Delta t)\}}{\|\hat{\boldsymbol{\omega}}\|^{2}}
$$

$$
\begin{gathered}
\Phi_{12}=[\hat{\boldsymbol{\omega}} \times] \frac{\{1-\cos ((\|\hat{\boldsymbol{\omega}}\| \Delta t)\}}{\|\hat{\boldsymbol{\omega}}\|^{2}}-I_{3 \times 3} \Delta t \\
-[\hat{\boldsymbol{\omega}} \times]^{2} \frac{\{\|\hat{\boldsymbol{\omega}}\| \Delta t-\sin (\|\hat{\boldsymbol{\omega}}\| \Delta t)\}}{\|\hat{\boldsymbol{\omega}}\|^{3}} \\
\Phi_{21}=0_{3 \times 3} \\
\Phi_{22}=I_{3 \times 3} \\
G_{k}=\left[\begin{array}{cc}
-I_{3 \times 3} & 0_{3 \times 3} \\
0_{3 \times 3} & I_{3 \times 3}
\end{array}\right]
\end{gathered}
$$

$$
Q_{k}=\left[\begin{array}{cc}
\left(\sigma_{v}^{2} \Delta t+\frac{1}{3} \sigma_{u}^{2} \Delta t^{3}\right) I_{3 \times 3} & \left(\frac{1}{2} \sigma_{u}^{2} \Delta t^{2}\right) I_{3 \times 3} \\
\left(\frac{1}{2} \sigma_{u}^{2} \Delta t^{2}\right) I_{3 \times 3} & \left(\sigma_{u}^{2} \Delta t\right) I_{3 \times 3}
\end{array}\right]
$$

A widely used model for a rate gyroscope is given by

$$
\begin{gathered}
\tilde{\boldsymbol{\omega}}(t)=\boldsymbol{\omega}(t)+\boldsymbol{\beta}(t)+\boldsymbol{\eta}_{v}(t) \\
\dot{\boldsymbol{\beta}}(t)=\boldsymbol{\eta}_{u}(t)
\end{gathered}
$$

where $\tilde{\boldsymbol{\omega}}(t)$ is the continuous time measured angular rate and $\boldsymbol{\eta}_{v}(t)$ and $\boldsymbol{\eta}_{u}(t)$ are independent mean zero Gaussian white-noise processes with

$$
\begin{aligned}
& E\left\{\boldsymbol{\eta}_{v}(t) \boldsymbol{\eta}_{v}^{T}(\tau)\right\}=I_{3 \times 3} \sigma_{v}^{2} \delta(t-\tau) \\
& E\left\{\boldsymbol{\eta}_{u}(t) \boldsymbol{\eta}_{u}^{T}(\tau)\right\}=I_{3 \times 3} \sigma_{u}^{2} \delta(t-\tau)
\end{aligned}
$$

where $\delta(t-\tau)$ is the Dirac delta function [38]. In practice, $\sigma_{v}$ and $\sigma_{u}$ are typically referred to as the angular random walk and rate random walk, respectively.

\section{A2 USQUE}

The USQUE is an implementation of the UF developed for attitude estimation [28]. Like the MEKF, it requires a three-axis rate gyroscope and at least one attitude sensor, and it maintains the quaternion norm constraint using the same multiplicative approach. In this section, the notation is largely the same as that used for the MEKF, such as $P$ representing state-error covariance, but the equations in this subsection are unique to the USQUE and should not be combined with the MEKF equations except where noted.

USQUE uses generalized Rodrigues parameters to define the attitude error vector as in Eq. (A21), where $a$ is a parameter from 0 to 1 and $f$ is a scale factor. This is analogous to Eq. (A2) but is an exact relationship and facilitates the use of any parameterization of the error quaternion [28]. $\delta \boldsymbol{p}$ is equivalent to the Gibb vector when $a=0$ and $f=1$. The authors of the USQUE use $f=2(a+1)$ such that $\|\delta \boldsymbol{p}\|=\vartheta$, where $\vartheta$ is the rotation angle of the attitude quaternions,

$$
\delta \boldsymbol{p} \equiv f\left[\delta \boldsymbol{\rho} /\left(a+\delta q_{4}\right]\right.
$$

The main difference between the UF and EKF lies in the manner in which Gaussian random variables are quantified and propagated through the dynamic model. In the EKF, the state-error covariance is propagated linearly via a first-order linearization of the nonlinear system. The UF quantifies the state distribution by using carefully chosen sample points, called sigma points. The sigma points completely capture the mean and covariance of the state and are propagated through the true nonlinear system [39]. Given an $n \times n$ state covariance matrix $P$, a set of $2 n \sigma$ points are generated from the 
columns of the matrices $\pm \sqrt{(n+\lambda) P}$, where $\sqrt{M}$ is shorthand notation for a matrix $Z$ such that $Z Z^{T}=M . \lambda$ is a scalar parameter that can be used to exploit knowledge about higher-order moments for the given distribution, if available [27]. For more details, see [28] and [39] and the references therein.

The procedure for attitude estimation with the USQUE is the following. The estimated quaternion, estimated state, and state-error covariance are first initialized to $\hat{\boldsymbol{q}}_{0}^{+}, \hat{\boldsymbol{x}}_{0}^{+}=\left[\begin{array}{ll}0_{1 \times 3} & \hat{\boldsymbol{\beta}}_{0}^{+T}\end{array}\right]^{T}$, and $P_{0}^{+}$, respectively. Next, the sigma points are calculated using Eqs. (A22) and (A23). The process covariance can be accounted for as in Eq. ( $\overline{\mathrm{A} 22)}$ because it is purely additive [39].tit The sigma points are partitioned as in Eq. (A24), where $\chi_{k}^{\delta p}$ is the attitude error portion and $\chi_{k}^{\beta}$ is the gyro bias portion. The corresponding error quaternions and $\sigma$ point quaternions are calculated with Eqs. (A25) and (A26), respectively. The quaternions are subsequently propagated using Eqs. (A27) and (A28), where $\Omega($ ) is given by Eq. (A11). The propagated error quaternions are then calculated with Eq. (A29), and the sigma points are propagated with Eqs. (A30) and (A31). The predicted state and state-error covariance can now be calculated using Eqs. (A32) and (A33). Using the predicted quaternion from Eq. (A27), the mean observation is calculated with Eqs. (A34) and (A $\overline{35}$ ). The output covariance, innovation covariance, and cross-correlation matrix are computed with Eqs. (A36-A38). The gain is then computed with Eq. (A39), and the state and error covariances are updated with Eqs. (A40-A42). The updated state is used to calculate the updated error quaternion with Eq. (A43), which is subsequenty used to update the attitude estimate with Eq. (A44). Finally, the attitude error vector $\delta \hat{\boldsymbol{p}}_{k+1}^{+}$is reset to zero for the next propagation. A detailed derivation and explanation of the USQUE can be found in [28] and the references therein,

$$
\begin{aligned}
& \chi_{k}(0)=\hat{\boldsymbol{x}}_{k}^{+} \\
& \chi_{k}(i)=\sigma_{k}(i)+\hat{x}_{k}^{+}, \quad i=112 \\
& \boldsymbol{\sigma}_{k} \leftarrow 2 n \text { columns from } \pm \sqrt{(n+\lambda)\left[P_{k}^{+}+Q_{k}\right]} \\
& Q_{k}=\left[\begin{array}{cc}
\left(\sigma_{v}^{2} \Delta t+\frac{1}{3} \sigma_{u}^{2} \Delta t^{3}\right) I_{3 \times 3} & -\left(\frac{1}{2} \sigma_{u}^{2} \Delta t^{2}\right) I_{3 \times 3} \\
-\left(\frac{1}{2} \sigma_{u}^{2} \Delta t^{2}\right) I_{3 \times 3} & \left(\sigma_{u}^{2} \Delta t\right) I_{3 \times 3}
\end{array}\right] \\
& \chi_{k}(i) \equiv\left[\begin{array}{c}
\chi_{k}^{\delta p}(i) \\
\chi_{k}^{\beta}(i)
\end{array}\right], \quad i=0,1,, 12 \\
& \delta \varrho_{k}^{+}(i)=f^{-1}\left[a+\delta q_{4_{k}}^{+}(i)\right] \chi_{k}^{\delta p}(i), \quad i=1,2,, 12 \\
& \delta q_{4_{k}}^{+}(i)=\frac{-a\left\|\chi_{k}^{\delta p}(i)\right\|^{2}+f \sqrt{f^{2}+\left(1-a^{2}\right)\left\|\chi_{k}^{\delta p}(i)\right\|^{2}}}{f^{2}+\left\|\chi_{k}^{\delta p}(i)\right\|^{2}}, \\
& i=1,2,, 12 \\
& \hat{\boldsymbol{q}}_{k}^{+}(0)=\hat{\boldsymbol{q}}_{k}^{+} \\
& \hat{\boldsymbol{q}}_{k}^{+}(i)=\delta \boldsymbol{q}_{k}^{+}(i) \otimes \hat{\boldsymbol{q}}_{k}^{+}, \quad i=1,2,, 12
\end{aligned}
$$

\footnotetext{
${ }^{\dagger}$ The authors of the USQUE derived a different treatment of $Q$ that approximates the integration of the process noise over the sampling interval, but this has been shown to have a negligible impact on the performance of the filter, so it is not used here [22,28].
}

$$
\begin{array}{r}
\hat{\boldsymbol{q}}_{k+1}^{-}(i)=\Omega\left[\hat{\boldsymbol{\omega}}_{k}^{+}(i)\right] \hat{\boldsymbol{q}}_{k}^{+}(i), \quad i=0,1,, 12 \\
\hat{\boldsymbol{\omega}}_{k}^{+}(i)=\tilde{\boldsymbol{\omega}}_{k}-\chi_{k}^{\beta}(i), \quad i=0,1,, 12
\end{array}
$$$$
\delta \boldsymbol{q}_{k+1}^{-}(i)=\hat{\boldsymbol{q}}_{k+1}^{-}(i) \otimes\left[\hat{\boldsymbol{q}}_{k+1}^{-}(0)\right]^{-1}, \quad i=0,1,, 12
$$$$
\chi_{k+1}^{\delta p}(0)=0
$$$$
\chi_{k+1}^{\delta p}(i)=f \frac{\delta \rho_{k+1}^{-}(i)}{a+\delta q_{4_{k+1}}^{-}(i)}, \quad i=1,2,, 12
$$$$
\chi_{k+1}^{\beta}(i)=\chi_{k}^{\beta}(i), \quad i=0,1,, 12
$$$$
\hat{\boldsymbol{x}}_{k+1}^{-}=\frac{1}{n+\lambda}\left\{\lambda \chi_{k+1}(0)+\frac{1}{2} \sum_{i=1}^{2 n} \chi_{k+1}(i)\right\}
$$$$
P_{k+1}^{-}=\frac{1}{n+\lambda}\left\{\lambda\left[\chi_{k+1}(0)-\hat{\boldsymbol{x}}_{k+1}^{-}\right]\left[\chi_{k+1}(0)-\hat{\boldsymbol{x}}_{k+1}^{-}\right]^{T}\right.
$$$$
\left.+\frac{1}{2} \sum_{i=1}^{2 n}\left[\chi_{k+1}(i)-\hat{\boldsymbol{x}}_{k+1}^{-}\right]\left[\chi_{k+1}(i)-\hat{\boldsymbol{x}}_{k+1}^{-}\right]^{T}\right\}+Q_{k}
$$

$$
\hat{\boldsymbol{y}}_{k+1}^{-}=\frac{1}{n+\lambda}\left\{\lambda \gamma_{k+1}(0)+\frac{1}{2} \sum_{i=1}^{2 n} \gamma_{k+1}(i)\right\}
$$

$$
\boldsymbol{\gamma}_{k+1}(i)=\left[\begin{array}{c}
A\left[\hat{\boldsymbol{q}}^{-}(i)\right] \boldsymbol{r}_{1} \\
\vdots \\
A\left[\hat{\boldsymbol{q}}^{-}(i)\right] \boldsymbol{r}_{N}
\end{array}\right]_{k+1}
$$

$$
\begin{gathered}
P_{k+1}^{y y}=\frac{1}{n+\lambda}\left\{\lambda\left[\gamma_{k+1}(0)-\hat{\boldsymbol{y}}_{k+1}^{-}\right]\left[\boldsymbol{\gamma}_{k+1}(0)-\hat{\boldsymbol{y}}_{k+1}^{-}\right]^{T}\right. \\
\left.+\frac{1}{2} \sum_{i=1}^{2 n}\left[\gamma_{k+1}(i)-\hat{\boldsymbol{y}}_{k+1}^{-}\right]\left[\gamma_{k+1}(i)-\hat{\boldsymbol{y}}_{k+1}^{-}\right]^{T}\right\}
\end{gathered}
$$

$$
P_{k+1}^{v v}=P_{k+1}^{y y}+R_{k+1}
$$

$$
\begin{gathered}
P_{k+1}^{x y}=\frac{1}{n+\lambda}\left\{\lambda\left[\chi_{k+1}(0)-\hat{\boldsymbol{x}}_{k+1}^{-}\right]\left[\gamma_{k+1}(0)-\hat{\boldsymbol{y}}_{k+1}\right]^{T}\right. \\
\left.+\frac{1}{2} \sum_{i=1}^{2 n}\left[\chi_{k+1}(i)-\hat{\boldsymbol{x}}_{k+1}^{-}\right]\left[\gamma_{k+1}(i)-\hat{\boldsymbol{y}}_{k+1}^{-}\right]^{T}\right\}
\end{gathered}
$$

$$
K_{k}=P_{k}^{x y}\left(P_{k}^{v v}\right)^{-1}
$$

$$
\hat{\boldsymbol{x}}_{k}^{+}=\hat{\boldsymbol{x}}_{k}^{-}+K_{k} \boldsymbol{v}_{k}
$$

$$
\boldsymbol{v}_{k} \equiv \tilde{\boldsymbol{y}}_{k}-\hat{\boldsymbol{y}}_{k}^{-}=\tilde{\boldsymbol{y}}_{k}-\boldsymbol{h}\left(\hat{\boldsymbol{x}}_{k}^{-}, k\right)
$$




$$
\begin{gathered}
P_{k}^{+}=P_{k}^{-}-K_{k} P_{k}^{v v} K_{k}^{T} \\
\delta \boldsymbol{\rho}_{k+1}^{+}=f^{-1}\left[a+\delta q_{4_{k+1}}^{+}\right] \delta \hat{\boldsymbol{p}}_{k+1}^{+} \\
\delta q_{4_{k+1}^{+}}^{+}=\frac{-a\left\|\delta \hat{\boldsymbol{p}}_{k+1}^{+}\right\|^{2}+f \sqrt{f^{2}+\left(1-a^{2}\right)\left\|\delta \boldsymbol{p}_{k+1}^{+}\right\|^{2}}}{f^{2}+\left\|\delta \boldsymbol{p}_{k+1}^{+}\right\|^{2}} \\
\hat{\boldsymbol{q}}_{k+1}^{+}=\delta \boldsymbol{q}_{k+1}^{+} \otimes \hat{\boldsymbol{q}}_{k+1}^{-}(0)
\end{gathered}
$$

\section{Acknowledgments}

This research is supported by the Department of Defense through a National Defense Science and Engineering Graduate fellowship. The Radio Aurora Explorer satellites were funded by the National Science Foundation. The authors thank Alex Sloboda and Alex Fox for providing feedback on this manuscript as well as to the entire Radio Aurora Explorer team for their support of the mission and this work. The authors also thank the anonymous reviews for their helpful feedback.

\section{References}

[1] Lerner, G. M., "Sun Sensors," Spacecraft Attitude Determination and Control, Kluwer, Dordrecht, The Netherlands, 1978, pp. 155-166.

[2] Dado, S., and Fischer, J., "Light Detectors," Modern Sensors Handbook, ISTE, Ltd., London, 2007, pp. 54-62.

[3] Tambo, T., Shibata, M., Mizuno, Y., and Yamauchi, T., "Search Method of Sun Using Fixed Five Photodiode Sensor," IEEJ Transactions on Sensors and Micromachines, Vol. 129, No. 2, 2009, pp. 53-59. doi:10.1541/ieejsmas.129.53

[4] Springmann, J. C., and Cutler, J. W., "Optimization of Directional Sensor Orientation with Application to Sun Sensing," Journal of Guidance, Control, and Dynamics, Vol. 37, No. 3, 2014, pp. 828-837. doi:10.2514/1.61468

[5] Ortega, P., Lopez-Rodriguez, G., Ricart, J., Dominguez, M., Castaner, L., Quero, J., Tarrida, C., Garcia, J., Reina, M., Gras, A., and Angulo, M., "A Miniaturized Two Axis Sun Sensor For Attitude Control of Nano-satellites," IEEE Sensors Journal, Vol. 10, No. 10, Oct. 2010, pp. $1623-1632$. doi:10.1109/JSEN.2010.2047104

[6] Wu, S.-F., and Steyn, W. H., "Modelling and In-Orbit Calibration Practice of a Miniature 2-Axis Analogue Sun Sensor," Aerospace Science and Technology, Vol. 6, No. 6, 2002, pp. 423-433. doi:10.1016/S1270-9638(02)01187-2

[7] Rufino, G., Grassi, M., and Rolfi, M., "Preliminary Calibration Results for a High-Precision CMOS Sun Sensor," Proceedings of the AIAA Guidance, Navigation, and Control Conference, AIAA Paper 20087319, 2008.

[8] Bhanderi, D. D. V., "Spacecraft Attitude Determination with Earth Albedo Corrected Sun Sensor Measurements," Ph.D. Thesis, Aalborg Univ., Aalborg, Denmark, Aug. 2005.

[9] Springmann, J. C., Sloboda, A. J., Klesh, A. T., Bennett, M. W., and Cutler, J. W., "The Attitude Determination System of the RAX Satellite," Acta Astronautica, Vol. 75, June-July 2012, pp. 120-135. doi:10.1016/j.actaastro.2012.02.001

[10] Taraba, M., Rayburn, C., Tsuda, A., and MacGillivray, C., "Boeing's CubeSat TestBed 1 Attitude Determination Design and On-Orbit Experience," Proceedings of the AIAA/USU Conference on Small Satellites, SSC Paper SSC09-X-6, 2009.

[11] Ovchinnikov, M., Ilyin, A., Kupriynova, N., Penkov, V., and Selivanov, A., "Attitude Dynamics of the First Russian Nanosatellite TNS-0," Acta Astronautica, Vol. 61, Nos. 1-6, 2007, pp. 277-285. doi:10.1016/j.actaastro.2007.01.006

[12] Allgeier, S. E., Mahin, M., and Fitz-Coy, N. G., "Design and Analysis of a Coarse Sun Sensor for Pico-Satellites," Proceedings of the AIAA Infotech at Aerospace Conference and Exhibit and AIAA Unmanned... Unlimited Conference, AIAA Paper 2009-1837, 2009.

[13] Springmann, J. C., Kempke, B. P., Cutler, J. W., and Bahcivan, H., "Initial Flight Results of the RAX-2 Satellite," Proceedings of the 26th Annual AIAA/USU Conference on Small Satellites, SSC Paper SSC12XI-5, 2012.
[14] Springmann, J. C., and Cutler, J. W., "Initial Attitude Analysis of the RAX Satellite," Advances in the Astronautical Sciences: Proceedings of the AAS/AIAA Astrodynamics Specialist Conference, AAS Paper 2011600, 2011.

[15] Springmann, J. C., "On-Orbit Calibration of Photodiodes for Attitude Determination," Proceedings of the USU/AIAA Small Satellite Conference, AAS Paper 2013-600, 2013.

[16] Shuster, M. D., Pitone, D. S., and Bierman, G. J., "Batch Estimation of Spacecraft Sensor Alignments I. Relative Alignment Estimation," Journal of Astronautical Sciences, Vol. 39, No. 4, Oct.-Dec. 1991, pp. 519-546.

[17] Shuster, M. D., and Pitone, D. S., "Batch Estimation of Spacecraft Sensor Alignments II. Absolute Alignment Estimation," Journal of Astronautical Sciences, Vol. 39, No. 4, Oct.-Dec. 1991, pp. 547-571.

[18] Alonso, R., and Shuster, M. D., "Complete Linear Attitude-Independent Magnetometer Calibration," Journal of Astronautical Sciences, Vol. 50, No. 4, 2003, pp. 477-490.

[19] Foster, C. C., and Elkaim, G. H., "Extension of a Two-Step Calibration Methodology to Include Nonorthogonal Sensor Axes," IEEE Transactions on Aerospace and Electronic Systems, Vol. 44, No. 3, 2008, pp. 1070-1078. doi:10.1109/TAES.2008.4655364

[20] Springmann, J. C., and Cutler, J. W., "Attitude-Independent Magnetometer Calibration with Time-Varying Bias," Journal of Guidance, Control, and Dynamics, Vol. 35, No. 4, 2012, pp. 1080-1088. doi: $10.2514 / 1.56726$

[21] Pittelkau, M. E., "Kalman Filtering for Spacecraft System Alignment Calibration," Journal of Guidance, Control, and Dynamics, Vol. 24, No. 6, 2001, pp. 1187-1195. doi: $10.2514 / 2.4834$

[22] Lai, K.-L., Crassidis, J., and Harman, R., "In-Space Spacecraft Alignment Calibration Using the Unscented Filter," AIAA Guidance, Navigation, and Control Conference, AIAA Paper 20035563, 2003.

[23] Duan, F., Liu, J., Li, R., and Li, D., "Real-Time Attitude-Independent Sun-Sensor/Magnetometer Calibration Algorithm for Micro-Satellite," Proceedings of the SPIE - The International Society for Optical Engineering, Vol. 5985, SPIE, Wuhan, China, 2005, pp. 598548-1598548-5.

[24] Reid, D., "Calibration of the Milstar Attitude Determination System," Proceedings of the 1997 American Control Conference, Vol. 1, Albuquerque, NM, 1997, pp. 557-563.

[25] Lefferts, E., Markley, F., and Shuster, M., "Kalman Filtering for Spacecraft Attitude Estimation," Journal of Guidance, Control, and Dynamics, Vol. 5, No. 5, 1982, pp. 417-429. doi: $10.2514 / 3.56190$

[26] Crassidis, J. L., Markley, F. L., and Cheng, Y., "Survey of Nonlinear Attitude Estimation Methods," Journal of Guidance, Control, and Dynamics, Vol. 30, No. 1, 2007, pp. 12-28. doi: $10.2514 / 1.22452$

[27] Julier, S. J., Uhlmann, J. K., and Durrant-Whyte, H. F., "A New Method for the Nonliear Transformation of Means and Covariances in Filters and Estimators," IEEE Transactions on Automatic Control, Vol. AC-45, No. 3, 2000, pp. 477-482. doi: $10.1109 / 9.847726$

[28] Crassidis, J. L., and Markley, F. L., "Unscented Filtering for Spacecraft Attitude Estimation," Journal of Guidance, Control, and Dynamics, Vol. 26, No. 4, 2003, pp. 536-542. doi: $10.2514 / 2.5102$

[29] Markley, F. L., "Attitude Error Representations for Kalman Filtering," Journal of Guidance, Control, and Dynamics, Vol. 26, No. 2, 2003, pp. 311-317. doi: $10.2514 / 2.5048$

[30] Bhanderi, D. D. V., "Modeling Earth Albedo Currents on Sun Sensors for Improved Vector Observation," Proceedings of the AIAA Guidance, Navigation, and Control Conference, AIAA Paper 20066592, 2006.

[31] Bhanderi, D. D. V., and Bak, T., "Modeling Earth Albedo for Satellites in Earth Orbit," Proceedings of the AIAA Guidance, Navigation, and Control Conference, AIAA Paper 2005-6465, 2005.

[32] Cutler, J. W., and Bahcivan, H., "The Radio Aurora Explorer-A Mission Overview," Journal of Spacecraft and Rockets, Vol. 51, No. 1, 2014, pp. 39-47. doi:10.2514/1.A32436

[33] Cutler, J. W., Springmann, J. C., Spangelo, S., and Bahcivan, H., "Initial Flight Assessment of the Radio Aurora Explorer," AIAA/USU Small Satellite Conference, Utah State Univ., Logan, UT, 2011.

[34] Psiaki, M. L., Martel, F., and Pal, P., "Three-Axis Attitude Determination via Kalman Filtering of Magnetometer Data," Journal of 
Guidance, Control, and Dynamics, Vol. 13, No. 3, 1990, pp. 506-514. doi: $10.2514 / 3.25364$

[35] Burton, R., Rock, S., Springmann, J., and Cutler, J., "Online Attitude Determination of a Passively Magnetically Stabilized Spacecraft," $23 r d$ AAS/AIAA Spaceflight Mechanics Meeting, AAS Paper 2013-364, 2013.

[36] Crassidis, J. L., and Junkins, J. L., Optimal Estimation of Dynamic Systems, CRC Press, Boca Raton, FL, 2004, pp. 419-433.

[37] Appel, P., "Attitude Estimation from Magnetometer and Earth-AlbedoCorrected Coarse Sun Sensor Measurements," Acta Astronautica,
Vol. 56, Nos. 1-2, 2005, pp. 115-126.

doi:10.1016/j.actaastro.2004.09.001

[38] Farrenkopf, R. L., "Analytic Steady-State Accuracy Solutions for Two Common Spacecraft Attitude Estimators," Journal of Guidance, Control, and Dynamics, Vol. 1, No. 4, 1978, pp. 282-284. doi: $10.2514 / 3.55779$

[39] Wan, E. A., and van der Merwe, R., "The Unscented Kalman Filter," Kalman Filtering and Neural Networks, edited by Haykin, S., Wiley, New York, 2001, pp. 221-280. 